\title{
Simultaneously Recorded Single Units in the Frontal Cortex Go through Sequences of Discrete and Stable States in Monkeys Performing a Delayed Localization Task
}

\author{
Eyal Seidemann, ${ }^{1}$ Isaac Meilijson, ${ }^{1}$ Moshe Abeles, ${ }^{2}$ Hagai Bergman, ${ }^{2}$ and Eilon Vaadia' \\ 1 School of Mathermatical Sciences, Raymond and Beverly Sackler Faculty of Exact Sciences, and School of Medicine, Tel \\ Aviv University, Ramat Aviv 69978, Israel, and 2 School of Medicine and Center for Neural Computation, the Hebrew \\ University, Jerusalem 91120, Israel
}

To test whether spiking activity of six to eight simultaneously recorded neurons in the frontal cortex of a monkey can be characterized by a sequence of discrete and stable states, neuronal activity is analyzed by a hidden Markov model (HMM). Using the HMM method, we are able to detect distinct states of neuronal activity within which firing rates are approximately stationary. Transitions between states, as expressed by concomitant changes in the firing rates of several units, occur quite abruptly. The significance and consistency of the states are confirmed by comparison with simulated data.

The detected states are specific to a monkey's response in a delayed localization task, allowing correct prediction of the response in $\sim 90 \%$ of the trials. Similar predictive power is achieved by a model based simply on the response histograms
(PSTH) of the units. The two models reach this predictive ability with different time courses: the PSTH model gains predictive power with a higher rate in the first second of the delay, and the HMM gains predictive power with higher rate in the next $3 \mathrm{sec}$. In this later period, conventional methods such as the PSTH cannot detect any firing rate modulations, but the HMM successfully captures transitions between distinct states that are specific to the monkey's behavioral response and occur at highly variable times from trial to trial.

Our results suggest that neuronal activity in this later period is described best as transitions among distinct states that may reflect discrete steps in the monkey's mental processes.

Key words: rhesus monkey; spike-train analysis; hidden Markov model; state dynamics; frontal cortex; delayed localization
Introspectively, we conceive most of our mental activity as evolving serially in a sequence of mental states related to our memories, plans, and thoughts. A great deal of this mental activity, however, is not likely to be time-locked to specific sensory or motor events. This fact complicates the efforts of neurophysiologists to interpret neuronal activity that may be related sequentially to mental processes. For example, during the delay period of common behavioral tasks such as delayed localization or delayedmatch-to-sample, memories can be formed, attention can shift, and plans for actions can be made at varying points in time, yet most methods for analyzing neuronal activity, such as the peristimulus time histogram (PSTH), only permit detection of activity modulations that are locked in time to a stimulus onset or to execution of a movement.

If, indeed, neuronal activity in some cortical area goes through a sequence of states related to mental activity, transitions between these states are likely to occur at variable times and to manifest themselves by concomitant changes in the firing rates of many neurons, switching abruptly from one set of firing rates to another. Some models of neural networks, which are characterized by sequences of quasistable states (Hopfield, 1982; Amit, 1989),

\footnotetext{
Received May 12, 1995; revised Oct. 18, 1995; accepted Oct. 20, 1995

This work was supported in part by the United States-Israeli Binational Science Foundation. We thank Iris Halman, Yifat Prut, and Hamutal Slovin, who did the experimental work. E.S. thanks M. N. Shadlen, G. Horwitz, D. Feldman, and W. T. Newsome for helpful comments on this manuscript.

Correspondence should be addressed to Eyal Seidemann, Department of Neurobiology, Stanford University School of Medicine, Stanford, CA 94305.

Copyright $(1996$ Society for Neuroscience $0270-6474 / 96 / 160752-17 \$ 05.00 / 0$
}

provide additional motivation to search for stable states of neuronal activity. The frontal cortex is especially suitable for studying the existence of such neuronal states, because it has been implicated to play a major role in high-level processes such as working menory and planning of movements (for review, see Milner and Petrides, 1984; Fuster, 1985, 1988; Goldman-Rakic, 1987).

The goal of this paper, therefore, is twofold. The first goal is to introduce an implementation of the hidden Markov model (HMM) (Baum, 1970) that allows for the detection of firing-rate modulations that are not locked in time to external events. The second goal is to apply this model to the activity of several neurons that are recorded simultaneously from frontal cortical areas in monkeys performing a delayed localization task to test whether this activity can be characterized by sequences of discrete and stable neuronal states, which might be related to mental states.

Under the HMM, neuronal activity is assumed to be in one of a small number of states that give rise to specific firing rates of the recorded units; the sequence of states is assumed to be governed by a time-homogeneous Markov chain. These states are referred to as "hidden" because they are not observed directly but are assumed to govern the observed spike trains. We estimate the HMM parameters and systematically study the firing rate modulations that are associated with transitions between states. In addition, we study the specificity of the detected states to the monkey's behavioral responses by comparing the predictive power of the HMM to that of a method based on the PSTH.

The results of this work suggest that two types of underlying dynamics characterize the neuronal activity in the frontal cortex. The early period after a presentation of a visual stimulus (first 
The GO Mode

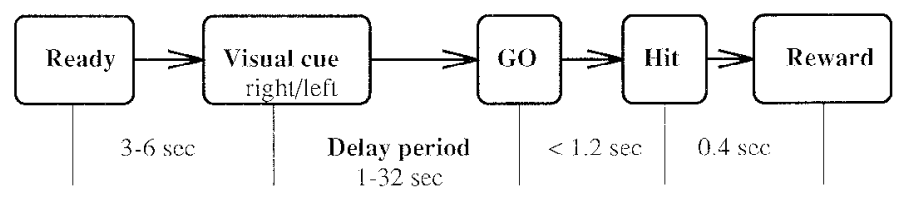

The No Go Mode

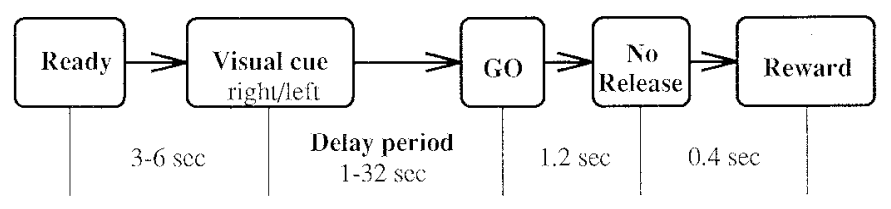

Figure 1. The sequence of behavioral events in the delayed localization task. In the GO mode, the monkey starts a trial by pressing a central startof-trial key. Then a ready light turns on and after 3-6 sec a visual stimulus appears either from the right or from the left. After a random delay of 1-32 sec, during which the monkey has to press the central start-of-trial key, a change in the color of the ready light serves as a $G O$ signal, atter which the monkey has to leave the central key and touch the cue light within $1.2 \mathrm{sec}$. The NO-GO mode procecds exactly like the GO mode, except that at the GO signal the monkey has to refrain from responding for at least $1.2 \mathrm{sec}$ to get the reward.

$1-1.5 \mathrm{sec}$ ) is marked by large modulations of firing rates, which are time-locked to the appearance of the stimulus. The later period is characterized by distinct states of neuronal activity that occur at highly variable times, yet are specific to the monkey's behavior. These states, therefore, might reflect some of the monkey's internal states occurring during the delay period.

Partial results of this research were presented in Seidemann et al. (1994) and Abeles et al. (1995).

\section{MATERIALS AND METHODS}

\section{Behavioral task}

Two female rhesus monkeys were trained to perform a delayed localization task with two behavioral modes, GO and NO-GO (Fig. 1). In the GO mode the monkey started a trial by pressing a central start-of-trial key. Then, a ready light turned on and after $3-6$ sec a visual stimulus appeared for $200 \mathrm{msec}$ either to the right or to the left of the ready light. The monkey had to memorize the location of the visual cue while continuously pressing the start-of-trial key during a random delay $(1,2,4,8,16$, or 32 $\mathrm{sec}$ ). A change in the color and intensity of the ready light served as a GO signal, after which the monkey had to relcase the start-of-trial key within $0.6 \mathrm{sec}$ and touch the cuc light within another $0.6 \mathrm{sec}$. If the monkey released the start-of-trial key before the appearance of the GO signal, the trial was aborted. Correct response was reinforced by $0.2-0.3 \mathrm{ml}$ of juice.

The NO-GO mode proceeded exactly like the GO mode, except that the monkey had to ignore the GO signal and keep pressing on the central start-of-trial key for at least $1.2 \mathrm{sec}$ after the GO signal to get the reward. Thus, the GO/NO-GO task allows one to study the way in which the same sensory information is processed under different behavioral demands. After the execution of four successful trials of one mode, all of the lights turned on for 3-4 sec; this served as a sign for the monkey to switch from one behavioral mode to the other.

After the monkey reached a suceess level of $>80 \%$, the monkey was anesthetized under aseptic conditions and a stainless stecl chamber was implanted above its frontal cortex. The recording started after a recovery period and repeated practice.

Single-unit activity was recorded from around the dorsomedial bank of the arcuate sulcus. In previous studies, we found that $>40 \%$ of the units in this area modulate their firing rates in relation to onc or several aspects of a similar task. In addition, it was shown that cooling and local injections of dopamine antagonists in this area caused deficits in the ability of monkeys to execute similar delayed localization tasks (Fuster and Baucr, 1974; Sawaguchi et al., 1994).
The treatment of the monkeys was in accordance with National Institutes of Health and Hebrew University regulations.

\section{Recording}

Spiking activity was recorded by a circular array of six glass-covered tungsten microclectrodes $(0.5-1.0 \mathrm{~mm}$ apart $)$, which were connected to a micromanipulator that allowed their indepcindent up and down movement. The recorded signal was filtered in the range of $0.2-8.0 \mathrm{kH}$. and sorted by multispike detectors. In the recording from monkey $\mathrm{B}$, two detectors based on principal components (Abeles, 19\%) and four window discriminators (Slope/Height window discriminators, Fredrick Haer \& Co. Cat. No. 74-60-1, Brunswick, ME) were used. In the recording from monkey $\mathrm{C}$, six detcctors based on template matching [Multi Spike Detector (MSD), Alpha-Omega Engineering, Nazareth, Israel] were used. The sorters, which were controlled interactively during the experiment, allowed separation of up to three single units from each electrode. The overall maximal number of simultaneously recorded units wals 16 . Typically, one or two well isolated units were recorded from each clectrode. Horizontal cye movements were recorded by electro-oculogram electrodes. The times of occurrence of spikes and behavioral events were measured with 1 msec resolution and stored on disk for subsequent analysis.

\section{Wata set}

Four recording sessions in which at least six units were well separated from each other and stable for at least 200 trials were chosen for analysis. We analyzed the neuronal activity in the first $4 \mathrm{sec}$ of the delay period after the visual cue in the trials in which the delay period was at leasi $4 \mathrm{sec}$. The analyzed data were classified into four behavioral conditions: the monkey performed a GO task with a visual stimulus from the right (GOr) or from the left (GOl), or a NO-GO task in which the stimulus was from the right (NGr) or from the left (NGl).

\section{The principles of the HMM}

This section gives a short and intuitive introduction to the HMM. A more formal description of the model and the parameter estimation algorithm are given in $\Lambda$ ppendix ( 1 and 2)

Our implementation of the HMM is based on the following assumptions. (1) The neuronal activity is well characterized by a relatively small number of hidden states; at every moment in time, the system is in one of these states. (2) In cach hidden state, the neurons firc according to homogeneous Poisson process. This means that in any state the neurons fire with constant firing rate, whereas the precise iming of spikes is random. (3) Hidden states change as a time-homogeneous Markov chain. This implies that at any moment in time, the probability of making a transition from the current hidden state to any of the others is a constant that depends only on these two states.

Figure $2 a$ shows a diagrammatic representation of an HMM based on the activity of six units with three hidden states, represented by boxes. Each state displays its profile of firing rates for the six recorded units. At any moment in time, the system is assimed 10 he in one of these three states. Therefore, the current hidden state determines the firing rates of the units at that lime step. After any time step, there is a certain probability of staying at the same state or making a transition to any of the other states. These probabilitics are indicated by the arrows from cach state to itself and to the other states.

For any given data sct of neuronal activity and a predelemined number of states, the Baum-Welch algorithm (Baum, 1970) may be applied to estimate the most likely HMM parameters: the profles of the firing rates in each state and the transition probabilities between states. The algorithm starts from an initial guess, similar to the one represented in Figure $2 a$, and iteratively finds parameters under which the likelihood of the data is higher.

Figure $2 b$ shows a diagrammatic representation of the HMM param eters, estimated for a data set that includes the spiking activity of 6 units in 93 GOr trials (presented as a raster display in Fig. $3 a$ ). This data set was fitted adequatcly woll by an HMM with six hidden states (see next section for further discussion regarding the number of hidden states). The profile of firing rates that characterizes cach state is indicated within the corresponding box. Typically, different states give rise to different firingrate profiles. For example, the firing rates of units 2 , 4 , and 6 are much lower in state 2 than in state 1 . A reduction in the firing rates of these units, therefore, might indicate a transition from state 1 to state 2.

As in Figure $2 a$, the arrows indicate the probability of staying at the same state and the probability of transition from one state to another at 
a.

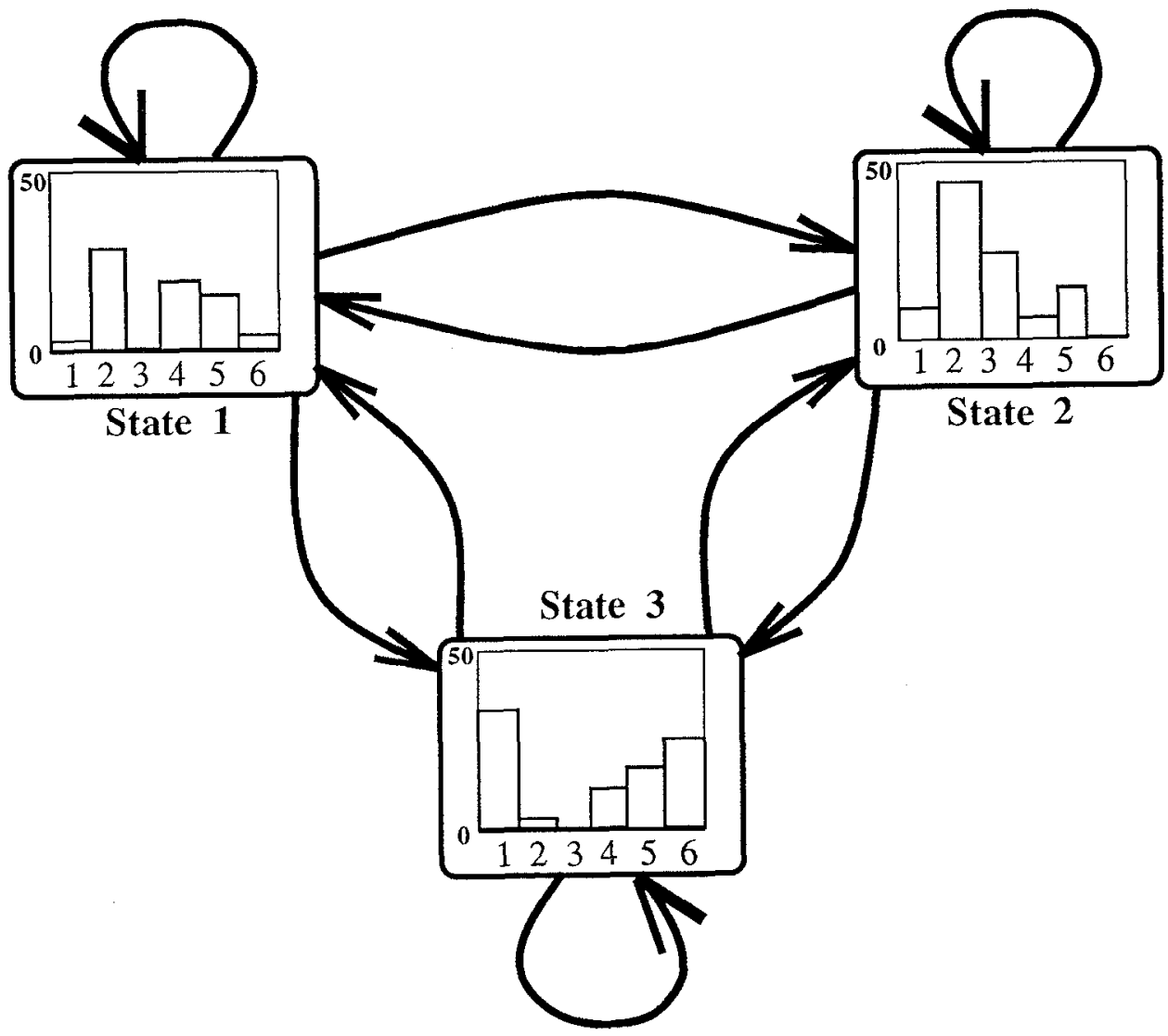

b.

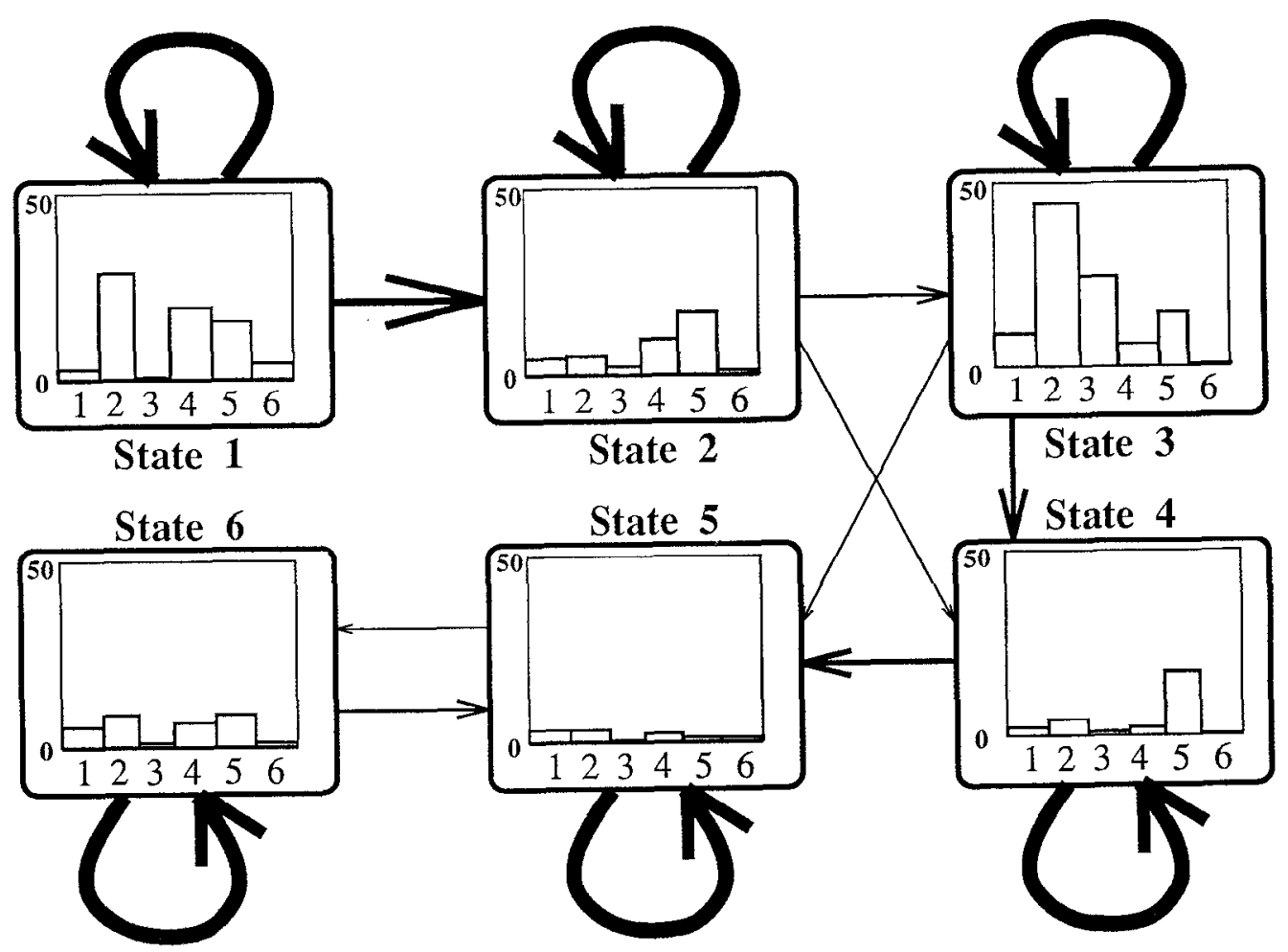

Figure 2. Diagrammatic representations of the HMM. a, An example of an HMM with three hidden states, represented by boxes. Each box displays the profile of firing rates of six units that characterize the corresponding state. The probabilities of transitions between states are represented by arrows, and the thickness of the arrow corresponds to the probability value. The arrow from each state to itself corresponds to the probability of staying at that state at any time step. $b$, HMM parameters that were estimated for the data set presented in Figure 3 . This data set was fitted by an HMM with six hidden at any time step. $b$, HMa a state to itself, because at 1 msec time-step transition probabilities are typically $10^{-2}-10^{-3}$ times lower than the sojourn probability. 
any time step. In this representation, the thickness of the arrow corresponds to the probability of transition. 'Iypically, most of the possible transitions have a very low probability $\left(<10^{-4}\right)$ and therefore are not shown. The probability of transition from most hidden states is concentrated mainly in a transition probability to one other state. One can see that initially the system is directional in time, will eventually reach state 5 and, thereafter, will alternate between states 5 and 6 .

Using a time step of $1 \mathrm{msec}$, which is smaller by an order of magnitude from the typical sojourn time within states, causes transition probabilities that are typically much smaller than the probability of staying at the same state (the arrows from each state to itself). The probability of staying in the same state at any time step [the sojourn probability, $P(i, i)$ ] determines the average sojourn time in state $i$ in a simple way: under the HMM assumptions, the sojourn times are distributed geometrically, and the average sojourn time in state $i$ is $1 /[1-\mathbf{P}(i, i)]$. For example, a probability $0.99 / \mathrm{msec}$ of staying at the same state (i.e., a probability of $0.01 / \mathrm{msec}$ of making a transition to some other state) corresponds to an average sojourn time of $100 \mathrm{msec}$. In this example, state 1 has the shortest average sojourn time (160 msec), whereas state 5 has the longest $(1420 \mathrm{msec})$.

After estimating the HMM parameters for a given data set, it is easy to calculate two quantities of interest. (1) For each trial, we can calculate the probability of each hidden state at every time step. These probabilities allow us to estimate the most likely sequence of hidden states behind each trial. (2) Given several different HMMs (for the different behavioral conditions), we can calculate the likelihood that the spike train of any given trial was produced by any of these models. This allows us to predict the behavioral condition as that under which a particular spike train is most likely.

\section{The estimation of the HMM parameters: practical considerations}

The HMM parameters were estimated separately for the trials of each of the fuur behavioral conditions by the Baum-Welch algorithm. The data set subjected to the estimation included only trials in which the monkey performed the task correctly. To apply the algorithm to the data, we first determine the best values of the number of hidden states and the initial probability vector (probability of starting at any state at time step one). The Baum-Welch algorithm, given these constraints, finds the maximum likelihood estimates of the transition probabilities between states and the firing rates of the units in each hidden state. The algorithm starts from an initial guess of these parameters and iteratively finds parameters under which the likelihood of the data is higher. The next sections explain our methods for choosing the number of hidden states, the initial probability vector, and the initial guess of the transition probabilitics and firing ratcs.

Initial probability vector. The probability of starting at any one of the hidden states at time step one had only a minor effect on the model into which the algorithm converged and on the average likelihood of the data. We tried two types of initial probability vectors: an equal probability of starting at any state and a probability of 1.0 to start at state 1 . Even when we started with equal probabilities, one state dominated the first period and the model parameters into which we converged were similar (other than shuffling state numbers). We therefore used a probability of 1.0 of starting at state 1 throughout our analysis. We note that our data set starts at the onset of the visual cue, whereas the actual response of the units starts after a delay of $50-150$ msec. Thus, state 1 is likely to represent the background activity before the visual response.

Number of hidden states. Obviously, changing the number of hidden states produced changes in the estimated parametcrs. Howcver, when we increased the number of states beyond six to eight states, the probability of transition into the additional states was close to zero (see Results regarding the distribution of transition times for an example). In three recording sessions, adding states beyond six, and in the fourth session adding states beyond eight, did not change the likelihood of the data significantly. Because our data are sparse and because the HMM allowed the prediction of the monkey's behavioral response quite accurately with six to eight states, we used an HMM with six hidden states in three analyzed sessions and eight states in the fourth recording session. We return to the issue of the number of hidden states in the Discussion.

Initial guess of transition probabilities and firing rates. Even for a given number of hidden states and initial probability vectors, the likelihood function may have multiple local maxima and, therefore, the estimated parameters may depend on the initial guess of the transition probabilities and firing rates. There is no obvious way by which one could know whether the maximum of the likelihood function that was reached is local or global. To overcome this difficulty, we started the algorithm from different initial guesses and chose as our parameters the ones under which the data had the highest likelihood.

We did not attempt to make any quantitative analysis or exhaustive search to test for the effect of the initial guess on the estimated HMM parameter. Our observation, however, was that for guesses with sojourn probabilities close to 1.0 , the algorithm converged to essentially the same states, independently of the first guesses of firing rates and transition probabilities. When the algorithm started with a guess in which the sojourn probabilities were $<0.9$ (corresponding to an average sojourn time of $<10 \mathrm{mscc}$ ), the averagc likclihood of the data under the estimated parameters was significantly lower. We therefore used throughout our analysis a guess with high sojourn probabilities (on the order of $0.95-0.999)$.

\section{RESULTS}

\section{Single-trial activity versus average response}

Figure $3 a$ presents the raster display of six units that were recorded simultaneously during one recording session. The neuronal activity in 93 trials in which the behavioral mode was GO and the visual cue appeared on the right (GOr trials) is shown. Each line in the raster display represents the spike train of a single unit during one trial, where each spike is represented by a dot. The trials are shown, one on top of the other in their chronological order, aligned to the onset of the visual cue. The time interval illustrated includes the first $4 \mathrm{sec}$ of the delay.

These ncurons clearly modulate their firing rates in the first second after the presentation of the visual cue, but later in the trial no responses that appear at a consistent time can be observed. Therefore, the changes in the average firing rates of the units, which are depicted in the PSTH (Fig. $3 b$ ), are restricted to the early period of the delay.

During the delay period, the monkey may plan an arm movement toward the location of the visual stimulus and hold this plan in register while attending to the GO signal, among other cognitive processes. Such cognitive processes, however, may not be tightly time-locked to the onset of the visual cue and may have variable time courses from trial to trial. Can we observe any additional firing rate modulations that occur at variable times during the delay and that, therefore, may reflect memory or planning related processes?

Figure $4, a$ and $b$ (bottom traces), shows in more detail the spiking activity in two single trials taken from this raster display. When the spiking activity in single trials is examined, it becomes clear that there is more going on than what can be seen in the PSTH. Units modulate their firing rates even in the later part of the trial. Furthermore, units tend to modulate their firing rates concomitantly. Intuitively, one might think that the increase in the activity of units 1,2, and 3 after $1.1 \mathrm{sec}$ (Fig. 4a, bottom trace) and the sharp decrease in their firing rates after 2.6 sec reflect transitions between states of neuronal activity. Similarly, in the second trial (Fig. $4 b$, bottom trace), units 1,2,3, and 4 increase their firing rates simultaneously at $450 \mathrm{msec}$ and then decrease it at 600 msec-or after $\sim 2.8 \mathrm{sec}$ units 1,3 , and 4 increase their firing rates concomitantly for half a second. Because these firing-rate modulations occur at different times in different trials, they are obscured by the PSTH of Figure 3.

To study these modulations systematically, one needs a method that detects firing-rate modulations even when they are not timelocked to external events. This can be achieved by the HMM.

\section{Single-trial activity under the HMM analysis}

Figure $2 b$ illustrates the HMM parameters that were estimated under the GOr trials that are presented as a raster in Figure 3. Once the most likely HMM parameters for a data set are found, 


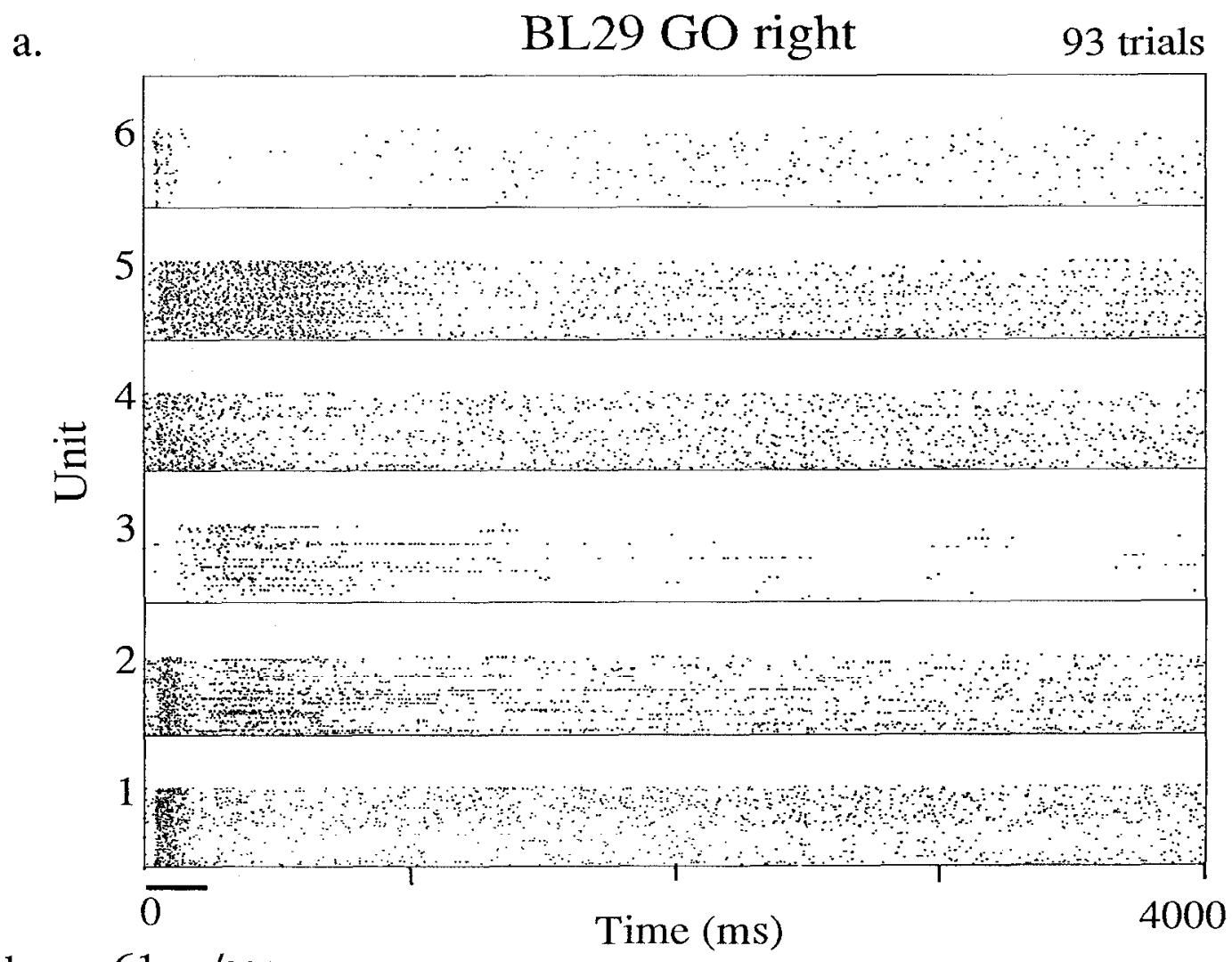

b. $\quad 61 \mathrm{sp} / \mathrm{sec}$

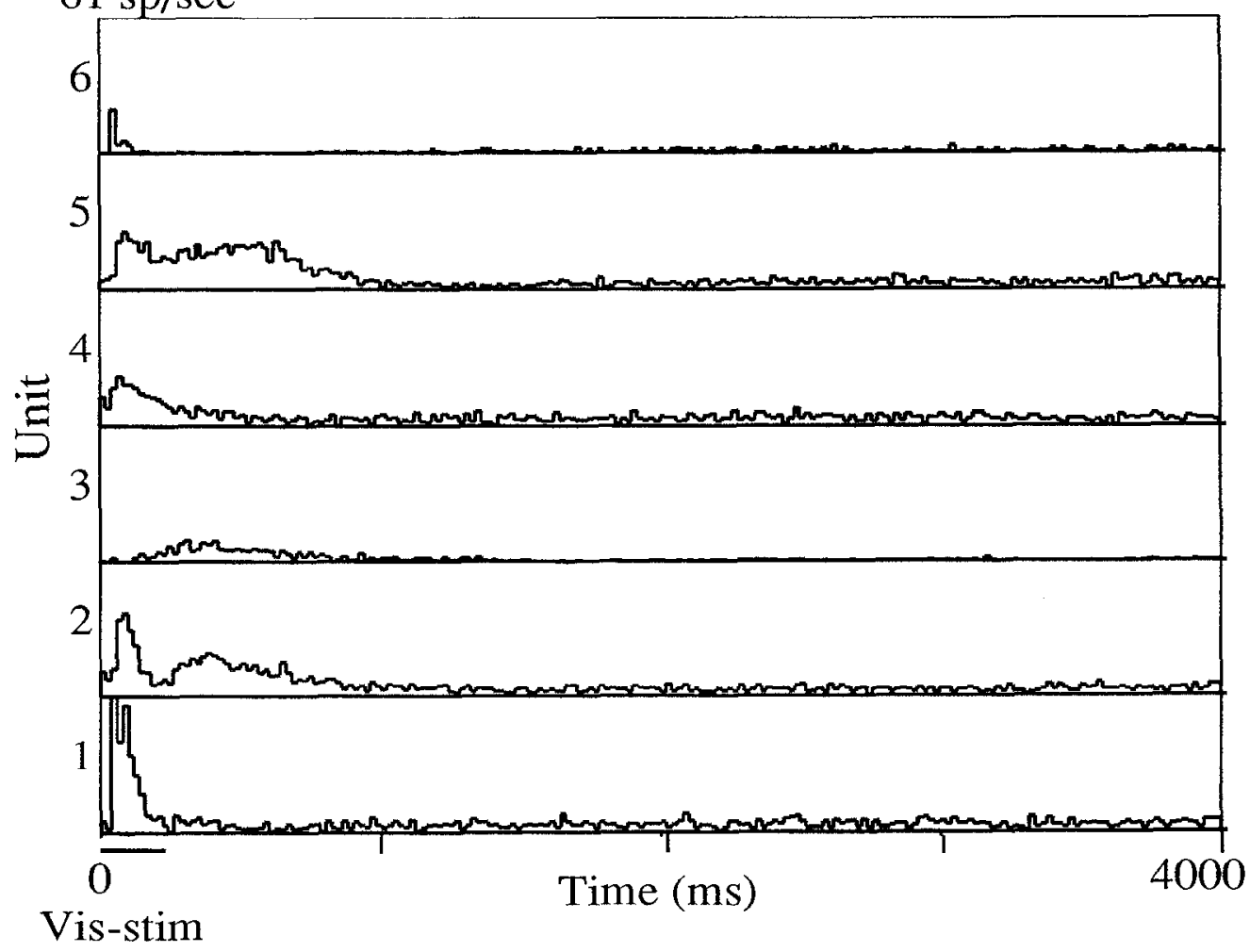

Figure 3. a, Raster display of 93 GOr trials from recording session BL29. The figure illustrates simultaneous activity of six well isolated units during 4 $\mathrm{sec}$ of the delay in trials with delay of at least $4 \mathrm{sec}$. Each trial is represented by a line, each spike by a dot. Trials are aligned to the presentation of the visual stimulus and arranged, one on top of the other, in their chronological order. $b$, A PSTH of the neuronal activity in $a$ in which the activity from all of the trials is summed and quantized into bins of 20 msec. 

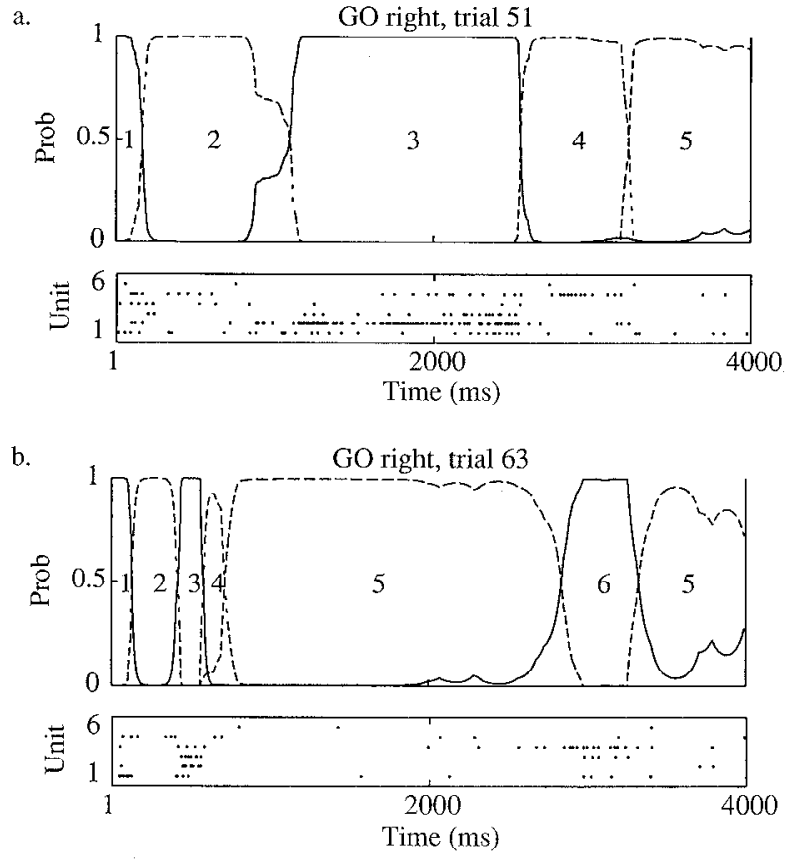

Figure 4. The spike trains of two GOr trials from recording session BL29 (taken from the raster display shown in Fig. 3) are shown during $4 \mathrm{sec}$ of delay in the bottom traces of $a$ and $b$. An HMM with six hidden states was estimated for all the GOr trials (Fig. $2 b$ ). For each trial, the computed probabilities of each one of the hidden states at every millisecond are shown above the trial's spike train (the number of the most probable hidden state also is indicated).

one can go back to the single trials and compute the probability of each one of the hidden states at any moment in time (Eq. 6, Appendix 1).

These probability functions are shown above the spike train of each trial in Figure 4 (top traces). In the first trial, the probability of state 1 is very high for $150 \mathrm{msec}$. Then there is a sharp transition and state 2 becomes highly probable, then states 3,4 , and 5 become most probable.

There are several interesting features in this figure. First, for most of the time there is one state that is much more probable than the others. Second, periods in which there is a transition from the one most probable state to the other are relatively short. Consequently, the estimated transitions between states, which are associated with concomitant changes in the firing rates of several units, appear to be quite sharp. One can also see that the HMM systematically finds the states that can be detected by eye in the spike trains. Finally, although these two trials have a similar sequence of hidden states, the timing of transitions between states is very different. State 3, for example, which starts at $1.1 \mathrm{sec}$ and lasts almost $1.5 \mathrm{sec}$ in the first trial (Fig. $4 a$ ), begins at 450 insec and lasts only $150 \mathrm{msec}$ in the second trial (Fig. $4 b$ ). The ability to accommodate such variable timing is one of the major advantages of the HMM, and makes it especially suitable for the analysis of neuronal processes with variable time course.

Figure 5 illustrates two NGr trials from the same recording session. Generally, the HMM parameters-firing rate vectors and transition probabilities-were quite different in the different behavioral conditions and across recording sessions. Because different recording sessions were conducted in different locations within the frontal cortex, the firing-rate vectors and transition probabilities did not show strong similarities across recording sessions. However, some similarities could be seen. Within each a.
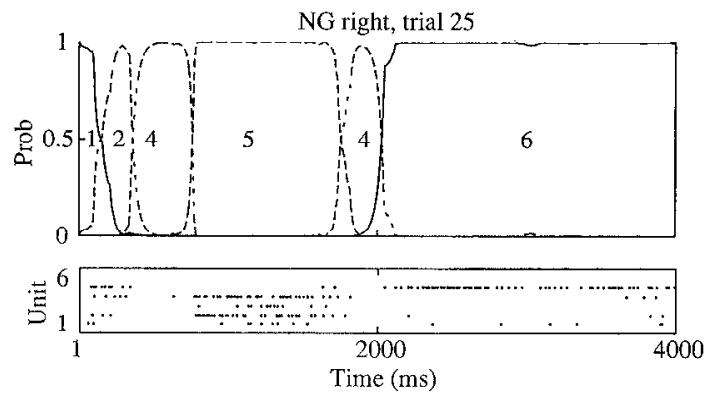

b.

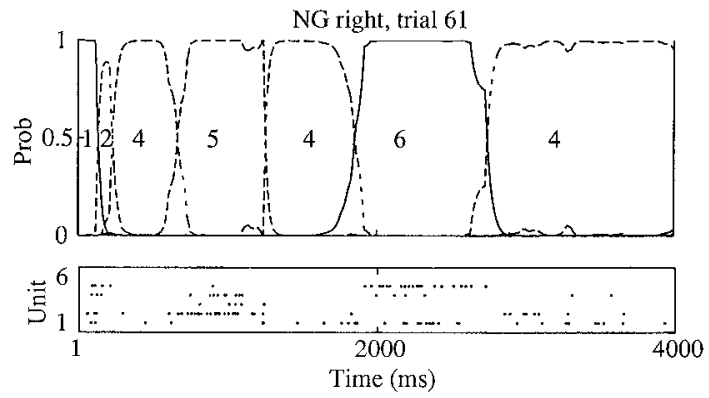

Figure 5. 'I wo NGr trials from recording session BL29. The probabilities of the six hidden states at every millisecond during $4 \mathrm{sec}$ of delay are plotted above the spike train of each trial.

recording session, the first two or three HMM states tend to be dominated by the visual response and, therefore, are relatively similar according to the direction of the visual cue (GOr is more similar to NGr, and GOl is more similar to NGl). These first states tend to have directional transition probabilities (as in the first 4 states in Fig. $2 b$ ). In the later part of the trials, the firing-rate vectors associated with the hidden states and the transition probabilities between these states tend to become similar in NGr and $\mathrm{NGl}$, whereas GOr and GOl states remain separated. An example of these later states in one recording session is presented in Figure 9. The transition probabilities between these later states show no directionality, and the later part of the trial is spent typically in transitions back and forth among as many as four states.

Thus, although the HMM parameters that were estimated for the NGr trials are quite different from those estimated for the GOr trials, the hidden states probability functions for these trials (Fig. 5) show the same general characteristics. Distinct states predominate, and the sharp transitions between them are associated with concomitant changes in the firing rates of several units.

\section{Estimated hidden states in simulated Poisson spike trains}

The probability functions that are presented in Figures 4 and 5 appear to support the existence of stationary states with sharp transitions between them. However, it can be argued that the HMM assumptions would force such states and transitions on any time-varying spike trains.

We would like to test whether the HMM could force such structure onto a data set in which units fire independently with deterministic firing rates that are time-locked to the beginning of the trial. Preferably, units in this data set would have the same average response as in the real data. The next section describes the method we used to estimate the average firing-rate modulations of the units. We then will describe the simulated data set that is based on these average firing rates. 


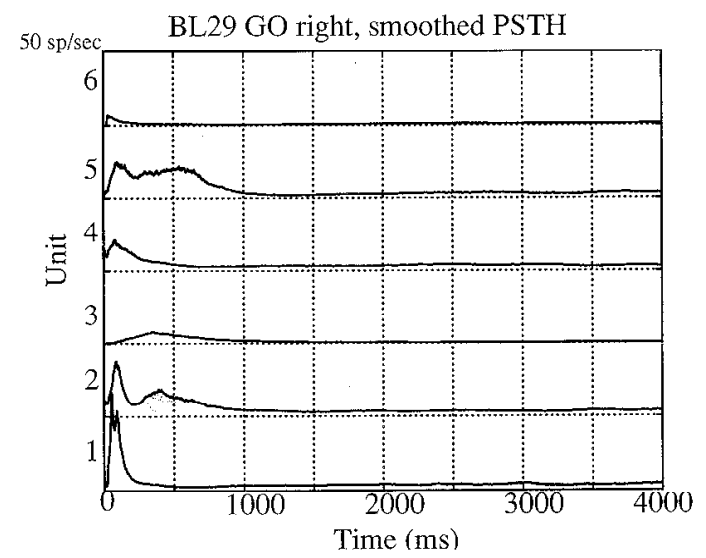

Figure 6. The estimated firing rate functions of the six recorded units under the NHPM assumptions. Instead of using a constant size bin (as in the PSTH of Fig. $3 b$ ), a window that contains a total of 100 spikes from all of the trials is used. The firing rate is estimated as the inverse of the length of the interval that contains the 100 spikes. This method smoothes the noisy fluctuations that can be seen in the PSTH and provides a constant relative error.

\section{Estimation of the average firing rates}

The PSTH in Figure $3 b$ depicts the modulations of the average firing rates that are time-locked to the onset of the visual stimulus in the GOr trials. In this PSTH, the spikes from all of the trials are summed and quantized into bins of $20 \mathrm{msec}$. The quantization of the neuronal activity into $20 \mathrm{msec}$ bins gives a rclativcly noisy estimate of the firing rates.

We would like to compute a continuous approximation of the underlying firing-rate $\lambda_{j}^{(i)}(t), 1 \leq t \leq 4000$, from the responses of unit $j$ during all trials of behavioral condition $i$. Because for each behavioral condition we had at most 100 trials and each neuron fires $\sim 1-50$ times/sec, a $1 \mathrm{msec}$ window will contain on average $0.1-5$ spikes/neuron (assuming 100 trials), hardly enough to estimate the firing rates. Assuming smoothness of the functions, we estimate $\lambda_{j}^{(i)}(t)$ as the average of $\lambda_{j}^{(i)}(s)$ for $s$ in the near vicinity of $t$, adaptively using a narrower window size in a period of high activity and a wider window under low activity. If $\lambda$ remained constant for some time, its estimate based on a window of $n \mathrm{msec}$ in $k$ trials would have mean $\lambda$ and SD $\sqrt{\lambda(1-\lambda) / n k} \simeq \sqrt{\lambda / n k}$. Hence, to have a constant relative error (SD/mean) of say $10 \%$, we should aim at $n k \lambda \simeq 100$. We apply this rule-of-thumb by estimating $\lambda_{j}^{(i)}(t)$ as the inverse of the length of the timc intcrval (symmetric around $t$ ), which contains a total of 100 spikes from neuron $j$ during all of the trials of behavioral condition $i$.

The results of this smoothing analysis, when applied to the GOr trials of recording session BL29, are shown in Figure 6. This procedure eliminates most of the noisy fluctuations in the PSTH (compare Fig. $3 b$ ) while capturing the significant features of the average response.

\section{HMM analysis of simulated nonhomogeneous Poisson data}

We used the firing-rate functions that were estimated from the PSTH to produce simulated data in which spikes are distributed as a nonhomogeneous Poisson process. Thus, simulated units fire spikes at rates that vary over time in a deterministic manner, but the exact timing of individual spikes is random. This procedure eliminates all of the non-time-locked and correlated firing-rate modulations of the real data while preserving the time-varying average firing rates.

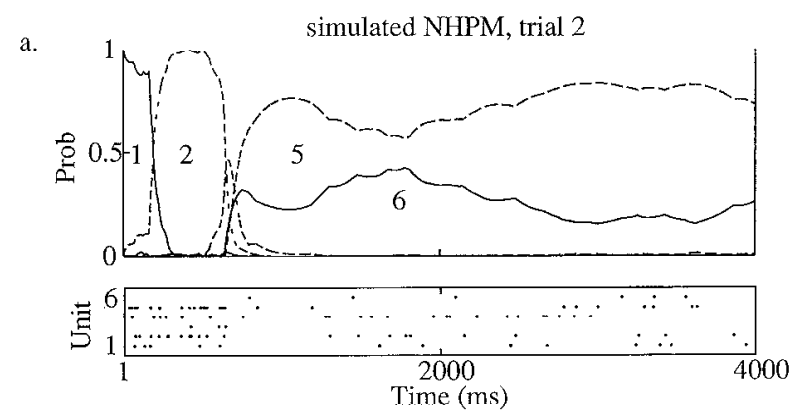

b.
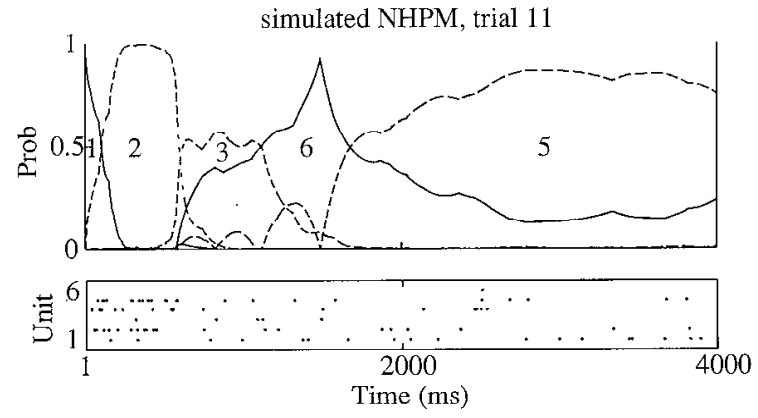

Figure 7. Two simulated trials that were generated using an NHPM with the firing-rate functions shown in Figure 6. The parameters of an HMM with six hidden states were estimated for the 93 simulated trials. The computed probabilities of each one of the states are shown above the spike train of the trials.

Table 1. Percentage of time with one dominant state in the experimental data and in simulated NHPM data

\begin{tabular}{lll} 
Recording session & Experimental data & Simulated data \\
\hline BL29 & 82.6 & 25.9 \\
BL21 & 62.0 & 24.9 \\
BL16 & 65.5 & 18.3 \\
CR43 & 65.1 & 16.4
\end{tabular}

For each recording session, simulated NHPM data were generated using the firing rates that were estimated for the recorded units. The same number of trials as in the experimental data set was generated in the simulation. The simulated data were analyzed under the HMM, and the percentage of time in which one of the hidden states had a probability that exceeded 0.8 was computed for the experimental neuronal data and for the simulated NHPM.

The same number of trials as in the real data set were simulated for each behavior in each recording session. The HMM parameters then were estimated for each of the simulated data. Figure 7 presents spike trains of two typical trials taken from the simulation of the GOr trials in recording session BL29.

The computed probabilities of the hidden states are shown above each simulated spike train. Although the transition probabilities during the first second resemble those in the real data, the probabilities in the rest of the trial differ dramatically. No clear states and sharp transitions between them are observed in this later period. Instead, one can see a competition between two alternative states.

Clearly, the HMM does not detect states with sharp transitions between them in the later period in which the simulated units fire with stationary firing rates. To get a better measure of this property, we compute the percentage of time in which one of the states has a probability $>0.8$. Table 1 illustrates the clear differences between the real and the simulated, nonhomogeneous Poisson data sets. 
Although in the real data one state predominates for $60-80 \%$ of the time, this happens only in $15-30 \%$ of the time in the simulated, nonhomogeneous Poisson data.

These results suggest that the states and sharp transitions detected in the real data under the HMM analysis reveal real properties of the neuronal activity and are not simply an artifact of the HMM-analysis method.

\section{Average firing rates around transition times}

In the examples presented in Figures 4 and 5, the transitions between the HMM states are associated with sharp and concomitant changes in the firing rates of several units, switching from one relatively stationary state to another. How typical are these transitions to the neuronal activity that we analyze, and how well are they detected by the HMM?

To answer these questions, we measure the averaged firing rates of the units around the timing of the detected transitions (the time in which there was an alteration in the most probable state). If sharp transitions between states are an artifact of the HMM assumptions and if the firing-rate modulations are actually slow and gradual in most of the trials, we would expect to see slow and gradual changes in the average firing rates around the detected transition times. Furthermore, if the firing-rate changes corresponding to the different units are not correlated significantly, we would expect the average firing-rate modulations of the different units around the detected transitions to have a different time course.

Figure $8 a$ presents the average firing rates of five units from recording session $\mathrm{BL} 29$, around the time of transitions between states $3,4,5$, and 6 ; the sixth unit was mostly silent in all of these states. We focus on the transitions between these states because they are associated with the later period of the delay. The spike counts are measured in windows of $25 \mathrm{msec}$ around the transition times; only time segments in which the first state was most probable for $100 \mathrm{msec}$ before the transition time, and the second state for $100 \mathrm{msec}$ after the transition, are included in this analysis.

The average firing rates before and after the transitions can be compared with their expected values under the HMM (presented in the corresponding boxes of Fig. $2 b$ ). For example, the expected firing rates of units 2,3 , and 5 in state 3 are 44,24 , and 14 spikes/sec, respectively, and 4, 1 , and 17 spikes/sec in state 4 , respectively. Consequently, units 2 and 3 show a major decrease in their firing rates in windows of $25 \mathrm{msec}$ before and after the transition time, whereas unit 5 maintains its firing rate. Similarly, the transition from state 4 to state 5 is associated with a decrease in the firing rates of units 2 and 5 and a slight increase in the firing rate of unit 1; the transition from state 5 to state 6 is associated with a concomitant increase in the firing rates of units 2,4 , and 5 , whereas the transition from state 6 back to state 5 shows the opposite modulations. Unit 2 modulates its firing rate in all of these transitions, demonstrating that the same unit can modulate its firing rate concomitantly with different neurons in different transitions.

Because of the relatively low firing rates, the limited number of trials, and the Poissonian nature of the data, these PSTHs are quite noisy. Still, most of the transitions are associated with sharp modulations of the average firing rates of several units, whereas the firing rates in the periods before or after the transition are relatively stationary. Furthermore, the firing-rate modulations of the different units appear to occur over similar time courses.

To test how well concomitant firing-rate modulations could be detected if the neuronal activity were in the form of an HMM, we
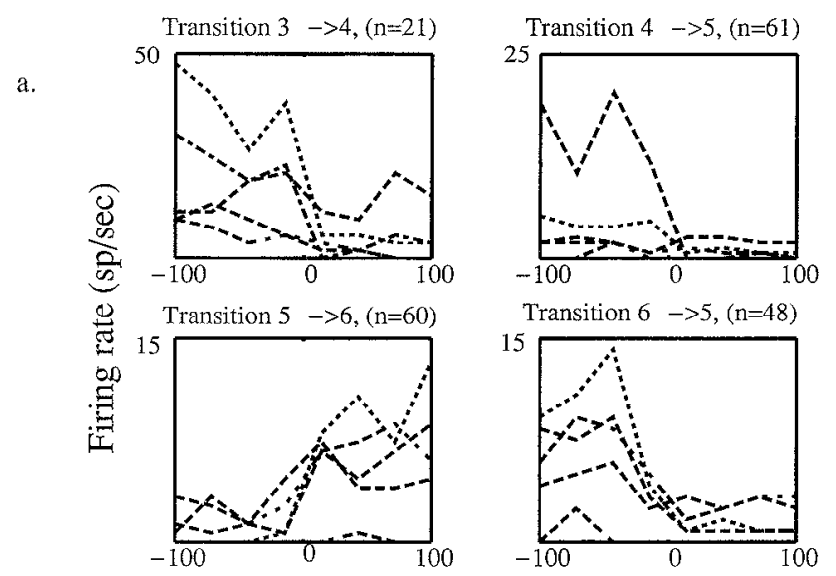

b.
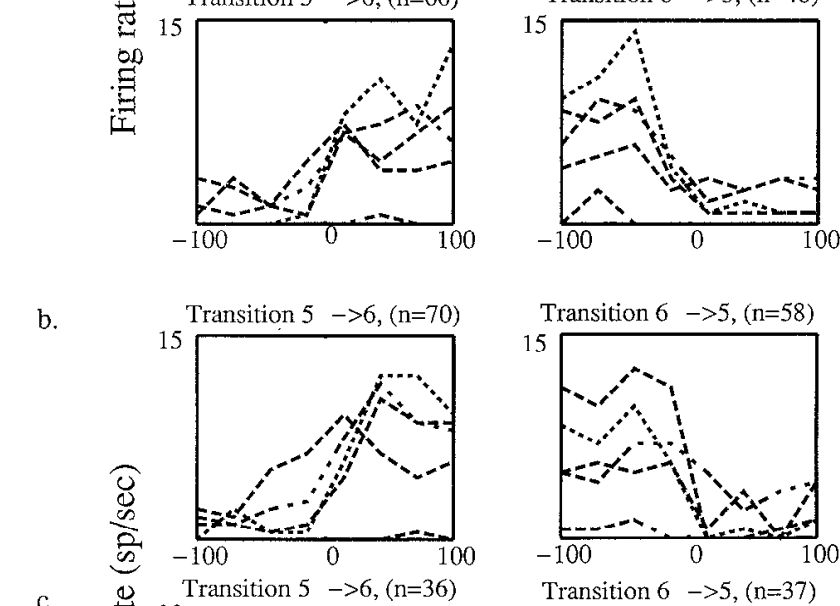

Transition $6->5,(\mathrm{n}=58)$

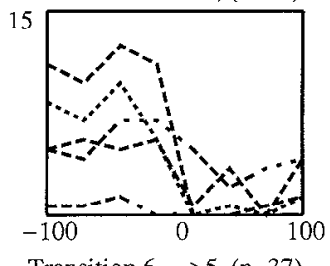

c.

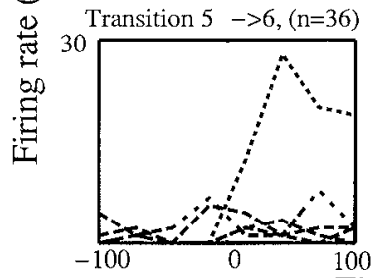

Transition $6 \rightarrow 5,(n=37)$

Time(ms)

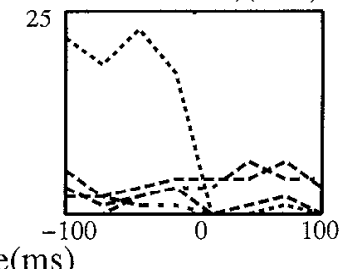

Figure 8. The PSTH of units 1-5 around transition times. Times in which the most probable state changed were considered transitions. The time segments that are analyzed include a period of $100 \mathrm{msec}$ before the transition, when the first state is the most probable, and $100 \mathrm{msec}$ after a transition, when the second state is the most probable. Spike counts are measured in $25 \mathrm{msec}$ windows. $a$, PSTH around transitions between states $3,4,5$, and 6 in recording session BL29 (GOr trials). $b$, PSTH around transitions between states 5 and 6 in a simulated HMM data set based on the parameters estimated for GOr trials of BL29 (Fig. 2b). c, PSTH around transitions between states 5 and 6 in the shuffled data set of BL29 (GOr trials) in which spike trains of different units were combined randomly across trials.

generated a simulated HMM data set using the parameter values estimated from the real data (Fig. $2 b$ ). The HMM parameters for this simulated data were re-estimated, and the average firing-rate modulations around transition times were measured. Figure $8 b$ presents the firing-rate modulations around transitions between states 5 and 6 in the simulated HMM data. These transitions appear to be similar to those detected in the real data (Fig. 8a), suggesting that the HMM-limited by the relatively low firing rates, the Poissonian nature of the spike trains, and the limited amount of data - cannot do much better in detecting such firingrate modulations.

This simulated HMM data provide another interesting measure: in this simulation, the actual state at any moment in time is known. Thus, the actual hidden state can be compared with the stale predicted by the HMM analysis. In $\sim 90 \%$ of the times, the most likely hidden state under the HMM analysis was the correct state, demonstrating that on simulated HMM data the HMM provides a very good estimate of the underlying sequence of 
states. Thus, if the HMM postulate is adequate, the empirical estimates we obtained can be considered reliable.

These results provide further support for the existence of stationary states of neuronal activity. The units clearly tend to modulate their firing rates together and abruptly. The HMM analysis appears to capture these stable states and to detect the sharp flipping between them reliably.

\section{Estimated hidden states in a shuffled data set}

To characterize further the role of concomitant firing-rate modulations in establishing the HMM states, we conducted HMM analysis of a shuffled data set. In principle, even if units fire independently, occasional concomitant firing-rate modulations could occur by chance and then be detected by the HMM across all trials of a given behavioral condition. To test whether the HMM could impose concomitant firing-rate modulations on a data set in which units modulate firing rates independently, we reorganized the data set by combining the responses of the units at random across the trials of the same behavioral condition. A typical trial in this shuffled data might include as "simultaneous" spike train the spike times of unit 1 in trial 10, unit 2 in trial 31 , unit 3 in trial 54, and so on. The advantage of the shuffled data set is that it maintains the intrinsic characteristics of the spiking activity (i.e., bursting activity) and eliminates correlated firing-rate modulations that are not timc-locked to the stimulus.

HMM parameters were estimated for the shuffled data set, and the average firing-rate modulations associated with the transitions between the states occurring in the later part of the delay were measured. Two examples of these PSTHs are given in Figure 8c. As expected, fring-rate modulations of mainly one unit are associated with these transitions. The HMM constructs the later hidden states according to the unit that shows the most marked changes from high to low firing rates. The average firing-rate modulations around the transition times in the shuffled data set (Fig. 8c) reflect, therefore, the lack of concomitant firing-rate modulations in this data set.

Figure 9 presents the HMM states that are associated with the later part of the trial under the four behavioral conditions in recording s ssion BL29. In the GO mode, the later part of the trial is associate $i$ with transitions back and forth between states 5 and 6 ; in the N-GO mode, this period is associated with transitions back and forth from state 4 to states 5 and 6 . Again, most of these states differ in the firing rates of several units. For example, in the NGr behivior the transition from state 4 to state 5 is associated with an iricrease in the firing rate of all units other than unit 6 . Similar transitions cannot be found when the shuffled data set is analyzed by the HMM. In the shuffled data, the detected states differ primarily in the firing rate of a single unit.

Thus, the absence of marked concomitant firing-rate modulations in the shuffled data indicates that coordinated firing-rate modulations are an important property of neuronal activity in the frontal cortex and are well captured by the HMM analysis.

\section{The distribution of transition times}

The trial-to-trial variability in the estimated time of transitions between hidden states is apparent in Figures 4 and 5. To investigate this variability, we registered the timing of a transition between states whenever an alteration in the most probable state occurred. For example, we register a transition from state 5 to state 4 at $1250 \mathrm{msec}$ in the first NGr trial (Fig. 5a) and at 1700 msec in the second trial (Fig. $5 b$ ). The distribution of transition times in all of the GOr and NGr trials of the same recording session (BL29) is shown in Figure 10, $a$ and $b$, respectively.
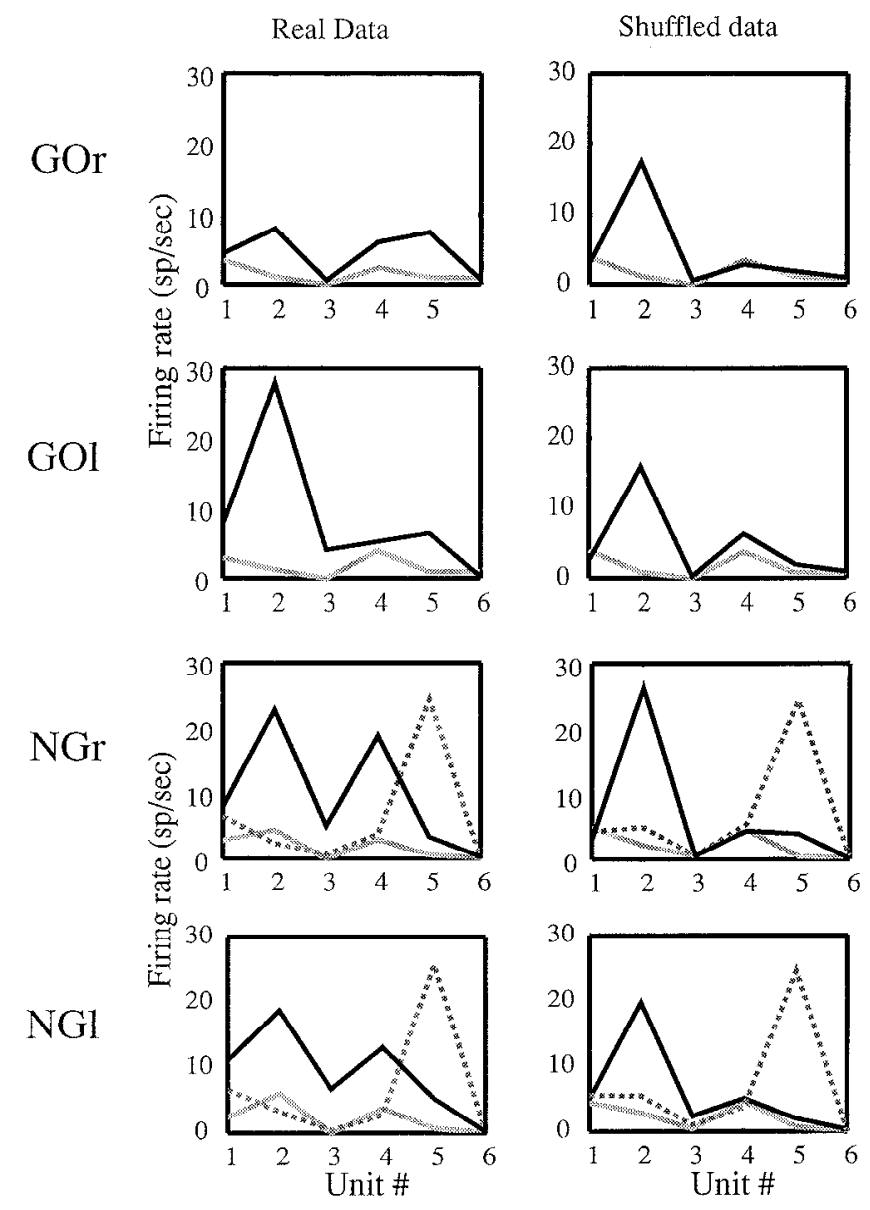

Figure 9. The firing-rate vectors of the HMM states that are associated with the later $2 \mathrm{sec}$ of the delay in the real and shuffled data from recording session BL29. For each behavioral condition, the real data set and shuffled data set in which responses of single units were combined randomly across trials were subjected scparately to estimation of the HMM parameters. The figure presents the firing rates of the six units in the later two states of GOr and GOl (state 5, gray; state 6, black) and the later three states in NGr and NGl (state 4, light gray; state 5, dashed dark gray; state 6 , black). The real data are presented on the left, and the shuffled data are presented on the right.

Most of the transitions between the first three or four hidden states occur in the first second after the onset of the visual cue. The timing of these early transitions appears to be centered around a typical value. For example, transitions from state 2 to state 4 occur typically $450 \mathrm{msec}$ after the onset of the visual cue in the GOr trials and at $350 \mathrm{msec}$ in the NGr trials. Transitions between states 5 and 6 appear to be distributed uniformly in the later $3 \mathrm{sec}$ of the GOr trials. In the NGr trials, this later period is characterized by transitions back and forth between state 4 and states 5 and 6 which, again, are distributed uniformly.

The distribution of transition times in the NGr trials provides an example for a redundant state. Almost all of the transitions from state 2 are to state 4 directly and not through state 3 (as in the two trials presented in Fig. 5). In this case, elimination of state 3 is not likely to affect the likelihood of the data.

These results suggest that, in the early period of the trials, the transitions between hidden states are relatively time-locked to the onset of the visual cue. Ilowever, transitions in the later $3 \mathrm{scc}$ or so are not time-locked and, accordingly, the changes in the firing rates underlying these transitions cannot be detected by any time-locked measure such as the PSTH. 

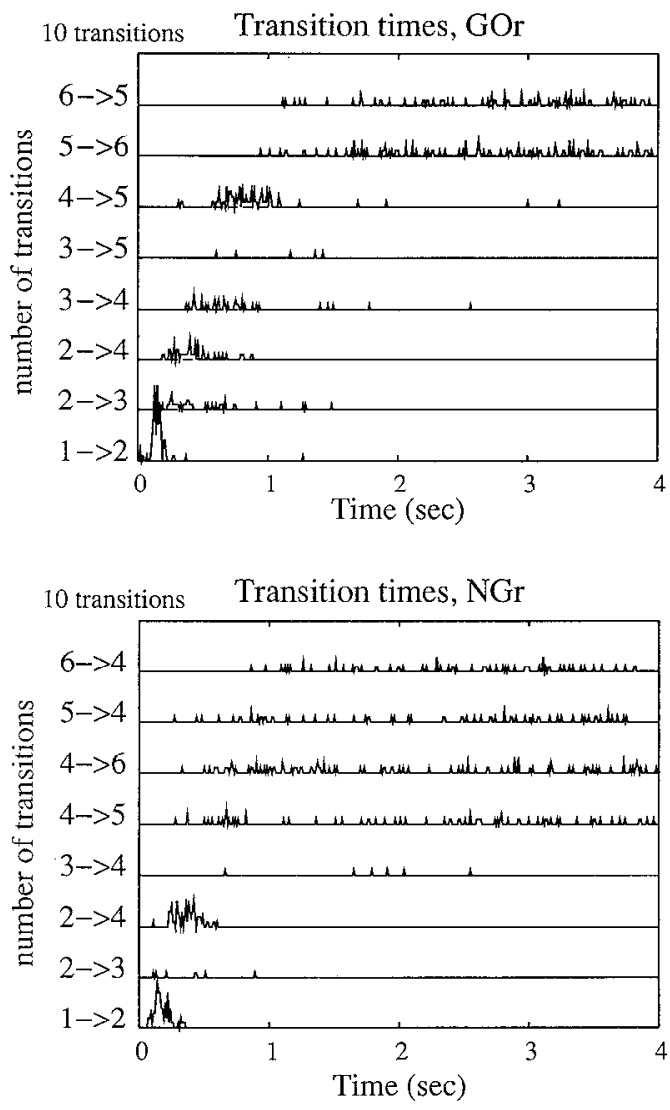

Figure 10. The distribution of transition times between hidden states in all of the GOr trials of BL29 (93 trials; top) and NGr trials (83 trials; bottom). Times in which the most probable state changed were considered transitions. The numbers of transitions observed over all of the trials in windows of $10 \mathrm{msec}$ are presented.

\section{Prediction of the monkey's response under the HMM}

So far, we have estimated the HMM parameters separately for trials of each one of the four different behavioral conditions: GOr, GOl, NGr, and NGl, analyzing only trials in which the monkey performed the task correctly.

We would like to know how specific the HMM parameters are to the monkey's behavioral conditions. Intuitively, the specificity should be measured by the ability of the model to predict the monkey's response from the spike train of a single trial. Thus, for each of the four behavioral conditions, we estimated the most likely model parameters for the observed data, as discussed previously. Figure 11 shows a diagram of the Bayesian prediction procedure that we used.

To predict the monkey's response, we simply compute the posterior probability that the spike train of a given trial was generated by the model of each of the four behaviors, and we choose as our prediction the model under which the spike train is most probable. The posterior probabilities are computed according to Equation 5 (for more details, see Appendix, 1).

The first step in this analysis is to estimate the model parameters separately for each behavioral condition. To compute the likelihood of a single trial, we performed the estimation of the HMM parameters omitting the spike train of this trial from the data set. We then computed the likelihood of that trial under the models of the four behavioral conditions. This jack-knife procedure was repeated for each and every trial in the data.

Table 2 displays the percentage of errors in the predictions of

\section{Prediction of the behavior}

Hidden Markov Model of:

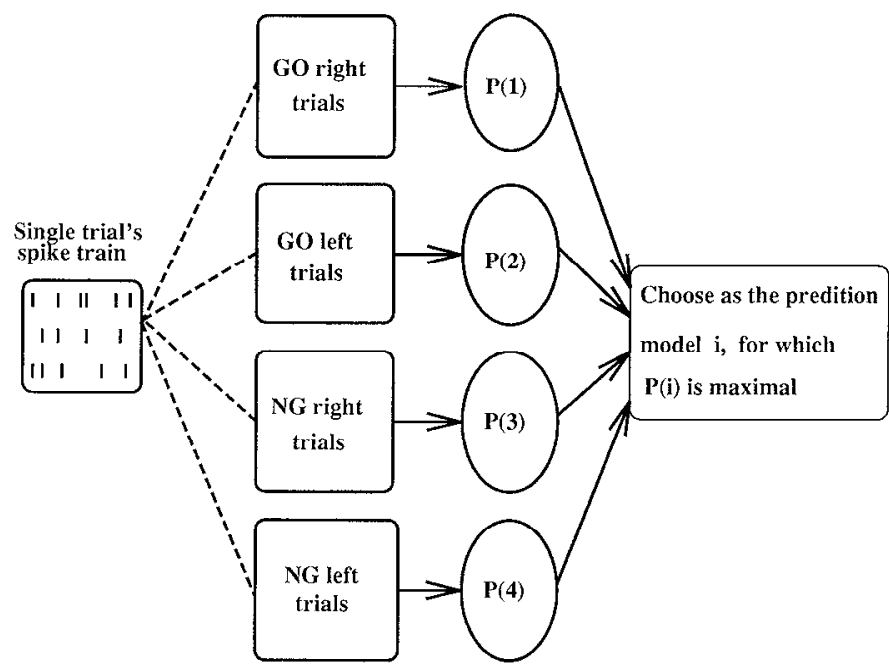

Figure 11. Illustration of the procedure used to predict the monkey's response. The probability of a spike train under each of the four models corresponding to the four behavioral conditions is computed first. The behavioral condition for which the model gives the maximal probability is chosen as the prediction.

Table 2. Percentage of errors in prediction of the behavioral condition under the HMM, NHPM, and HPM

\begin{tabular}{lrcr} 
& HMM & NHPM & HPM \\
\hline BL16 & & & \\
GO/NO-GO & 8.5 & 8.5 & 12.0 \\
GOr/GOl & 7.6 & 2.5 & 17.6 \\
NGr/NGl & 15.6 & 13.9 & 28.7 \\
BL29 & & & \\
GO/NO-GO & 7.6 & 8.8 & 17.5 \\
GOr/GOl & 5.7 & 4.0 & 20.7 \\
NGr/NGl & 14.8 & 10.1 & 38.1 \\
BL21 & & & \\
GO/NO-GO & 7.1 & 4.2 & 8.0 \\
GOr/GOl & 13.7 & 11.8 & 25.5 \\
NGr/NGl & 27.2 & 11.8 & 46.3 \\
CR43 & & & \\
GO/NO-GO & 15.8 & 11.6 & 28.6 \\
GOr/GOl & 2.7 & 0.7 & 18.1 \\
NGr/NGl & 16.0 & 17.3 & 34.6 \\
\hline
\end{tabular}

The procedure presented in Figure 11 was used to predict the monkey's behavioral condition in each one of the trials. For each recording session, the percentage of errors in prediction of $\mathrm{GO}$ versus $\mathrm{NO}-\mathrm{GO}, \mathrm{GOr}$ versus $\mathrm{GOl}$, and $\mathrm{NGr}$ versus $\mathrm{NGl}$ is presented separately.

the monkey's response under the HMM. To estimate the predictive power of the HMM, we separately compute the percentage of correct predictions of the behavioral mode ( $\mathrm{GO}$ vs $\mathrm{NO}-\mathrm{GO}$ ), the direction of the visual cue in the GO mode (GOr vs GOI), and the direction of the visual cue in the NO-GO mode (NGr vs NGl). For the GO versus NO-GO and GOr versus GOl predictions, the HMM predicts the monkey's response in $\sim 90 \%$ of the trials. The percentage of errors in the NGr versus NGl discrimination is larger than in the other two categories. Because the direction of the visual cue is irrelevant to the monkey's behavior in the 
NO-GO mode, it is not surprising that the activity of frontal neurons does not reflect the direction as clearly as in the GO trials.

The estimation of the model parameters for each behavioral condition was based on the trials in which the monkey performed the task correctly. We used the model derived from the correct trials to analyze trials in which the monkey made an error. Again, we used the measure of highest posterior probability to identify for each trial the most likely behavioral condition under which it was produced. Because of the sparsity of the monkey's errors $(<10 \%$ of the trials), all of the errors (e.g., $\mathrm{GO}$ behavior in a NO-GO trial and vice versa or an error in the localization of the cuc in the GO mode) wcre combined into one class in this analysis.

In 46 of 99 error trials, the behavior predicted by the HMM also differed from the expected behavior. Thus, the model predicted an alternative behavior (to the behavioral condition) in $\sim 50 \%$ of the trials in which the monkey made a mistake (in contrast to $10 \%$ in the correct trials).

\section{Comparison with standard methods}

The nonhomogeneous Poisson model

A common way to evaluate the neuronal response is via a PSTH. How well can the monkey's behavior in a single trial be predicted using the PSTHs related to the different behaviors?

To predict the monkey's behavior from the time-locked neuronal response that is measured in the PSTH, we assume that the neurons fire according to the time-varying firing-rate functions that are estimated using the smoothed PSTH method (see Results regarding estimation of the average firing rates). In addition, we assume that the precise timing of spikes is random as in a nonhomogeneous Poisson model (NHPM) and that spike trains of different units are independent.

Our model parameters in this case are the firing rate functions $\lambda_{j}^{(i)}(t)(1 \leq t \leq 4000)$ for each unit $j$ in any behavior $i$. After estimating these firing rate functions (see Fig. 6), we easily can compute the probability of any spike train under the model associated with each hehavior (Fq. 9; see Appendix 3 for more details). This allows us to predict the monkey's behavior using the same prediction procedure that was applied under the HMM (Fig. 11).

The predictive abilities of the NHPM and HMM are quite similar (Table 2). The NHPM predicts the behavioral mode (GO vs NO-GO) and the direction of the visual cue in the GO mode (GOr vs GO1) in $\sim 90 \%$ of the trials. As with the HMM, the number of confusions between NGr and NGl is relatively larger.

In 35 of the 99 trials in which the monkey made a mistake, the NHPM predicted an "alternative" behavior (to the behavioral condition). Hence, the ability of the NHPM to predict the monkey's mistakes is somewhat worse than that of the HMM (46/99).

\section{The homogeneous Poisson model}

An even simpler method for analyzing neuronal activity is the average number of spikes (or spike count) during some period after an external event. This method uses only the mean of the time-varying firing rates. We compared the predictive power of this method, which corresponds to a homogeneous Poisson model (HPM), to the predictive ability of the HMM and the NHPM. The HPM is very similar to the NHPM model, except that $\lambda_{j}^{(i)}(t)$ is assumed to be constant in $t$. We estimate $\lambda_{j}^{(i)}$ as the average number of spikes/msec that neuron $j$ fired in behavior $i$. The likelihood is calculated as in the NHPM model (Eq. 9). In agree- ment with previous works that demonstrated the valuable information in the firing-rate modulations (Optican and Richmond, 1987; Richmond and Optican, 1987, 1990a,b; McClurkin et al., 1991), the results presented in Table 2 indicate that the predictive power of the HPM is significantly worse than the predictive power of the NHPM and the HMM.

Our results indicate that predicting the behavioral condition using $4 \mathrm{sec}$ of delay activity is a relatively easy task. The posterior probabilities on which predictions are based turned out to be extremely close to 1 in the successful $90 \%$ of cases and to be grossly wrong in the others. In such clear-cut data, many models can provide high predictive power. For example, a neural network trained with back-propagation provides a similar predictive power as the HMM and the NHPM on the 4 sec of delay activity (F. Seidemann and N. Interator, unpublished observations). A comparison of the predictive power of the HMM and the NHPM under more difficult conditions is presented in the next section.

\section{The predictive power of the HMM and the NHPM in the later part of the delay}

The HMM and the NHPM predict the monkey's behavior with similar accuracy when $4 \mathrm{sec}$ of the delay are analyzed. To compare the predictive ability of the HMM and the NHPM in the later part of the delay, we excluded the first 1,2 , or $3 \mathrm{sec}$ of the delay and attempted to predict the monkey's behavior based on the later $1-4,2-4$, or 3-4 sec, respectively. 'The HMM and NHPM parameters were re-estimated for each truncated period, and the predictive power in each pcriod was compared with the predictive power obtained with the entire $4 \mathrm{sec}$ period (Table 2). The results of this analysis pooled over all recording sessions are shown in Figure $12 a$. The HMM and the NHPM were used to separately predict the behavioral mode (GO vs NO-GO; short dashed line), the direction of the visual cue in the GO mode (GOr vs GOl; solid line), and the direction of the visual cue in the NO-GO mode (NGr vs NGl; long dashed line).

In Figure 12, the percentage of errors is shown separately for the HMM (black lines) and the NHPM (gray lines) for the entire $4 \mathrm{sec}$ period and for the later 3,2, or $1 \mathrm{sec}$. For all three types of prediction categories, the HMM starts with slightly lower predictive power when the entire $4 \mathrm{sec}$ are analyzed. However, when the first second of the delay is excluded, the predictive power of the HMM exceeds that of the NHPM. Similarly, when the later 2-4 or 3-4 sec of the delay are analyzed, the HMM provides significantly higher predictive power than the NHPM.

These results indicate that in the later part of the delay the HMM provides additional predictive power by detecting firingrate modulations that are not time-locked to the appearance of the visual cue. It is important to note that although all of the analyzed PSTHs are flat in the later part of the delay, the average firing rates are typically different in the GO versus NO-GO mode trials and in the GOr versus GOl trials. Thus, the NHPM continued to gain some predictive power even in the later part of the trials, but at a slower rate than the HMM.

Part of the additional predictive power of the HMM might be achieved by the ability of the HMM to capture concomitant firing-rate modulations of several units. To test for the contribution of concomitant firing-rate modulations to the predictive power of the HMM, we analyzed the shuffled data set from one recording session and used the HMM to predict the behavioral condition in this data set. In the shuffled data, the spiking activity of different units is combined randomly across trials of the same 
a.

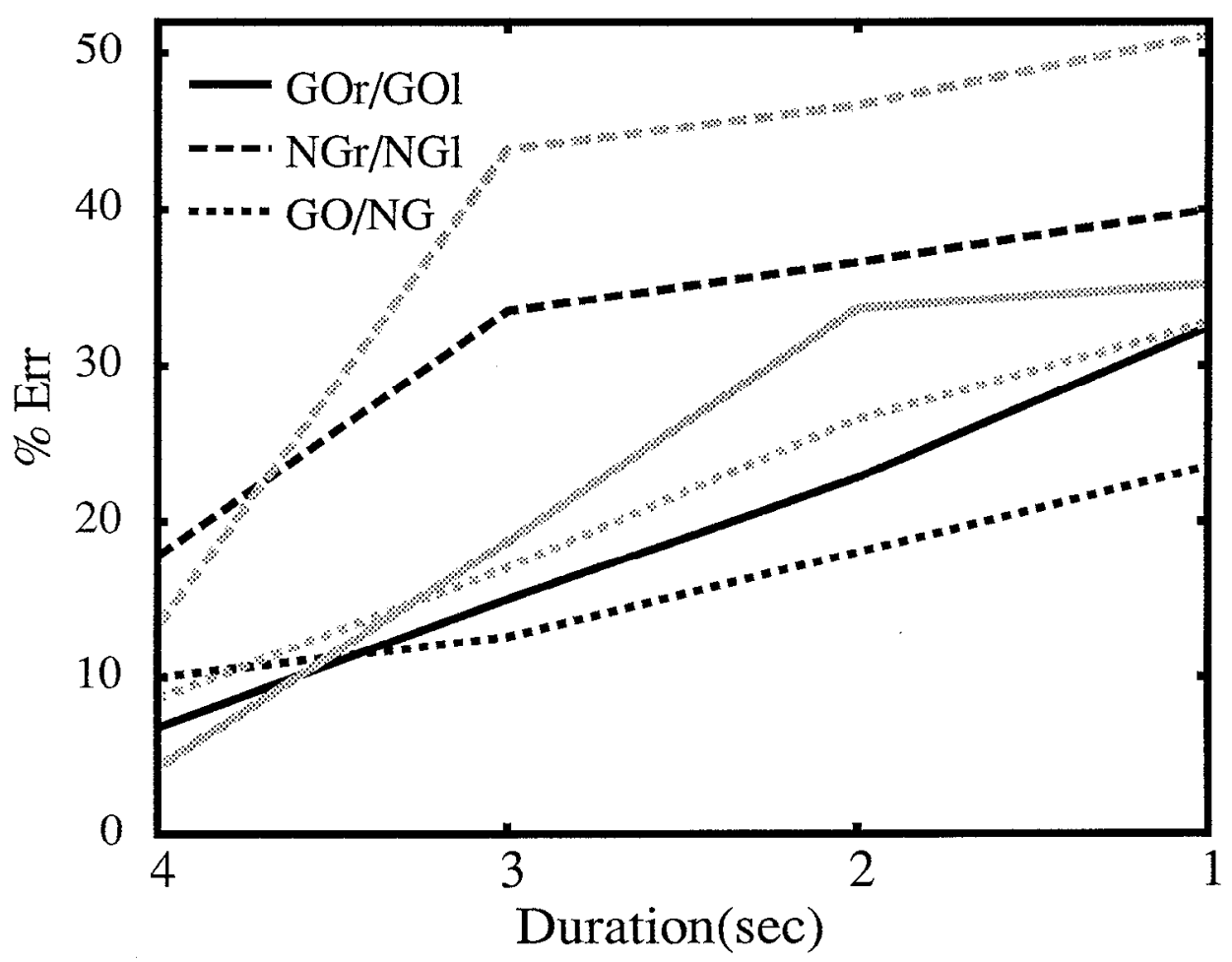

b.

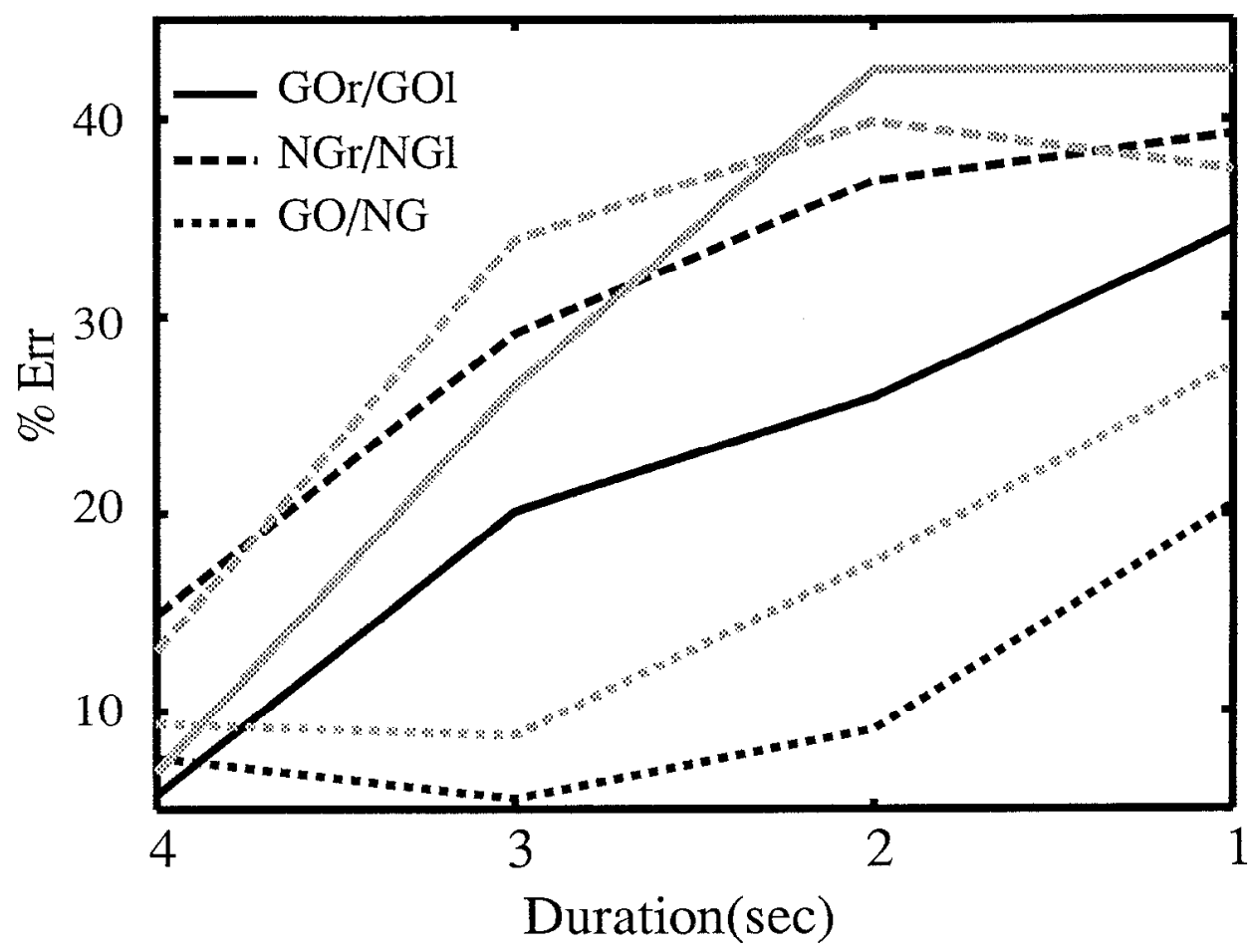

Figure 12. Percentage of errors in prediction of the monkey's behavioral condition based on the later part of the delay. The $x$ axis presents the duration of the analyzed period: 4 , the entire $4 \mathrm{sec}$ period; $3,1-4 \mathrm{sec} ; 2,2-4 \mathrm{sec} ; 1,3-4 \mathrm{sec}$. The figure presents separately errors in the prediction of GO versus NO-GO (short dashed line), GOr versus GOl (solid line), and NGr versus NGl (long dashed line). Chance level is 50\%. a, Comparison of the HMM (black lines) and the NHPM (gray lines). Data are combined from all recording sessions. $b$, Comparison of the percentage of errors in prediction of the behavioral condition under the HMM for the real (black lines) and shuffled (gray lines) data sets of recording session BL29. 
behavioral condition, so that any concomitant firing-rate modulations that might exist in the data and are not time-locked to the visual cue are eliminated. Figure $12 b$ shows the predictive ability of the HMM on the real (black lines) and shuffled (gray lines) data sets of recoding session BL29.

In this recording session, the HMM provides a slightly higher predictive power for the real data when the entire $4 \mathrm{sec}$ period is considered. The predictive power of the HMM falls more sharply for the shuffled data when the early part of the delay is excluded. For example, in the GO mode, when the monkey has to hold the direction of the visual cue in memory to perform the task correctly, the predictive power of the HMM in the later 2-4 sec falls dramatically when the shuffled data set is analyzed. These results suggest that some of the neuronal information regarding the direction of the visual cue and the behavioral mode is encoded by concomitant firing-rate modulations with variable timing.

Thus, although overall the two models predict the behavior with similar success, our results indicate that this predictive ability is achieved with a different time course. In the first period (1-1.5 $\mathrm{sec}$ ), which is dominated by the response to the visual cue, the NHPM has higher predictive power, suggesting that it characterizes the early neuronal response better. In the later part of the delay, when no time-locked firing-rate modulations are observed, the HMM gains more predictive power, indicating that it characterizes the neuronal activity better.

The lower predictive power of the HMM in the early period of the trials suggests that the early dynamics of the HMM require a higher multiplicity of hidden states than our sparse data are able to reliably detcet. The next section provides further support for this contention.

\section{Average firing rates under the HMM}

The early period after the presentation of the visual cue is characterized by relatively large modulations of the firing rates that are time-locked to the presentation of the visual cue. These changes are seen clearly in the PSTH of the GOr trials in recording session BL29 (see Fig. 3b).

We would like to measure how well the HMM captures the firing-rate modulations in the early period of the trials. To measure the average firing rates under the HMM, we first estimate the average probability of each one of the hidden states at any moment. We then use these probabilities to weight the firing rates of the units in the different states, obtaining an estimate of the average firing rates under the HMM. Figure 13 shows these average firing-rate functions for the six units in the GOr trials of recording session BL29. For comparison, Figure 6 shows the firing-rate functions of the same units when estimated under the NHPM.

Clearly, the HMM smears the fast modulations of the firing rates that are well captured by the NHPM in the early period of the trials. Unlike the smoothing procedure of the NHPM, the IIMM forces this continuous and complex response into a few discrete states. Hence, it is not surprising that the predictive power of the HMM is lower during this early period. We return to this point in the Discussion.

\section{Contribution of single units to the predictive ability}

The methods that have been introduced so far use information about the firing patterns of all of the analyzed neurons. In this section, we measure the contribution of individual units to the predictive ability of the entire recorded set. We compute the percentage of erroneous predictions when each of the units is
BL29 GO right, average firing rates under the HMM

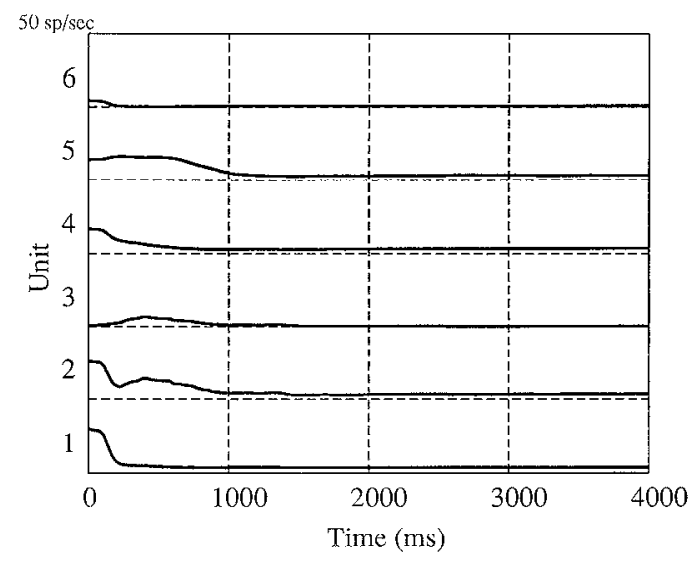

Figure 13. Average firing rates of six units in GOr trials of recording session BL29 under the HMM. The average probability of each one of the hidden states at any time step is used as a weighting vector for the firing rates in the different hidden states. For comparison, the average firing rates of the same units, estimated under the NHPM assumptions, are shown in Figure 6.

excluded from the data in its turn. To measure the predictive power of the HMM after elimination of a unit, we re-estimated the HMM parameters for the new data. Table 3 shows the effect of elimination of each one of the eight single units on the predictive ability of the HMM and the NHPM in recording session BL16.

Table 3 (top) presents the effect of elimination of a single unit on the predictive power of the HMM. The elimination of unit 4 produced maximal degradation in the prediction of GOr versus GOl. The elimination of units 1 and 4 produced large effects on the prediction of NGr versus NGl, whereas the prediction of the behavioral mode (GO vs NO-GO) was quite robust to elimination of any of the units.

The effect of eliminating single units on the predictive power of the NHPM is illustrated in Table 3 (bottom). As with the HMM analysis, the elimination of unit 4 produced maximal degradation in the prediction of the stimulus direction in the $\mathrm{GO}$ mode. Elimination of unit 1 caused maximal deteriorating effect in the prediction of the behavioral mode and in the prediction of NGr versus NGI. Under the NHPM, only a few units contribute information regarding the behavioral condition. The elimination of these informative units $(1,4$, or 8 in this session $)$ produced a much larger decrease in the predictive power of the NHPM than in the predictive power of the HMM. However, the other units $(2,3$, or 5-7) contributed very little to the predictive power of the NHPM and, thus, their elimination affected the predictive power minimally. The elimination of unit 7 even improved the predictive ability of the NHPM, suggesting that this unit contributes only noise.

The HMM is far less sensitive to elimination of single units in all of the recording sessions that were analyzed. These results can be attributed to the different ways the models treat the multiunit activity. The NHPM assumes that units are independent and does not take into account concomitant modulations of firing rates of several units. By treating all of the units as one entity, the HMM obtains additional information from the less informative units and, consequently, is less sensitive to the elimination of the informative units. 
Table 3. Percentage of errors in the prediction of the behavioral condition after elimination of single units

BL16 HMM

\begin{tabular}{lrrrrrrrr}
\hline Unit & 1 & 2 & 3 & 4 & 5 & 6 & \multicolumn{1}{c}{ All } \\
\hline GO/NG & 6.8 & 5.1 & 4.7 & 8.1 & 5.6 & 5.1 & 5.1 & 8.5 \\
GOr/GOl & 4.2 & 3.4 & 5.9 & 12.6 & 5.9 & 5.0 & 5.6 \\
NGr/NGl & 18.2 & 12.2 & 9.6 & 17.4 & 7.8 & 9.5 & 11.3 & 2.5 \\
\hline
\end{tabular}

BL16 NHPM

\begin{tabular}{lllllllll}
\hline Unit & 1 & 2 & 3 & 4 & 5 & 6 & 7 & 8 \\
\hline GO/NG & 30.3 & 5.1 & 6.8 & 27.8 & 7.7 & 6.4 & 5.6 & 22.2 \\
GOr/GOl & 2.5 & 3.4 & 1.7 & 47.9 & 2.5 & 2.5 & 0.8 & 2.5 \\
NGr/NGl & 32.2 & 9.6 & 9.6 & 26.9 & 7.8 & 8.7 & 6.9 & 8.7 \\
\hline
\end{tabular}

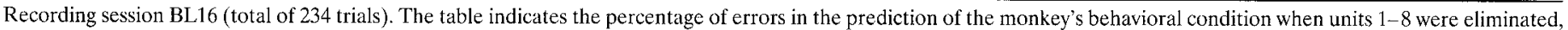

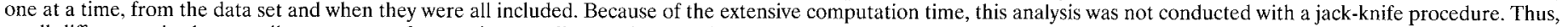
small differences in the overall percentage of errors in recording session BL16 can be seen between Tables 2 and 3 .

\section{DISCUSSION}

\section{The applicability of the HMM to neuronal activity}

A simple sensory-to-motor transformation is an inadequate explanation for all but the simplest reflexes. Rather, internal processes related to goals, plans, and other mental states intervene between sensory events and overt behavioral responses. Several electrophysiological studies in monkeys have demonstrated neuronal correlates of higher mental processes such as attention, intention, and memory (for review, see Posner and Petersen, 1990; Desimone et al., 1995). Analysis tonls that enable the researcher to follow neuronal activity that is not governed directly by external events are likely to play a major role in any quantitative study of these internal processes.

Thus, the first goal of this study was to introduce an implementation of the HMM that permits investigation of neuronal processes that are not locked in time to external events. We have shown that the HMM can be applied successfully to the activity of several neurons that were recorded simultaneously from frontal cortical areas of a monkey. The HMM detects firing-rate modulations that cannot be detected by measures such as the PSTH, thus providing important and useful information concerning the neuronal activity under study. Our results demonstrate the potential of the HMM in studies of neuronal processes that govern complex bchaviors.

We are aware of three other works that used HMM in analyzing neuronal activity. Deppisch et al. (1994) used the HMM to analyze oscillatory neuronal activity from cat visual cortex. Radons et al. (1994) used HMM to analyze single-unit activity obtained by 30 microelectrodes in area V1 of anesthetized monkeys. Gat and Tishby (1993) analyzed the same data set as in this study, but they used low tinte resolution ( $500 \mathrm{msec}$ windows shifted by $100 \mathrm{msec}$ ) and disregarded the stimuli and the monkey's behavior. We refer to some of the results obtained by the other methods in the following sections.

The HMM is likely to be a useful tool in the study of other neuronal processes with variable time course. In principle, the HMM analysis can be applied even when the response of only one unit is recorded. In this case, however, the important capacity of the HMM to detect concomitant firing-rate modulations would not be exploited. In addition, the number of states that would be detected in a single unit's data is likely to be lower.

In general, the number of hidden states that are supported by a data set is likely to depend on the number of recorded units, on the firing rates of the units, and on the amount of data that were collected. With our present data, we could detect six to eight distinct states of neuronal activity reliably. Although these particular numbers are not likely to have any biological significance and are merely a consequence of the amount and quality of the analyzed data, our results suggest that the obscrved states and the sharp transitions between them reflect real and interesting properties of the neuronal activity of the frontal cortex.

\section{Evidence for "state" dynamics}

Using the HMM analysis, we detected distinct states of neuronal activity. Essentially the same states and transition probabilities were estimated when the algorithm started from different initial guess parameters. The probability functions of the hidden states (Figs. 4 and 5) show that at most moments in time one of the states is predominant. The estimated transitions from one predominant state to the other are relatively abrupt, suggesting that the neuronal system switches quickly from one state to another. In contrast, in a simulated NHPM data set with the same average response (Fig. 7), single states did not predominate in general, and transitions between states were gradual.

Furthermore, in the real data, transitions between states are associated with concomitant changes in the firing rates of several units, switching from one relatively stationary state to the other (Fig. 8a). The significance of these concomitant firing-rate modulations was confirmed by analyzing a reorganized data set in which the activity of the units was combined randomly across trials (Fig. 8c). In this shuffled data set, the HMM did not find similar concomitant modulations in the later part of the delay (Fig. 9), demonstrating that the correlated nature of these firing-rate modulations is a genuine property of the analyzed activity.

Such concomitant firing-rate modulations within a small set of randomly selected neurons suggest that these neurons actually are embedded in a large network that switches from one stable configuration to another. Additional evidence for this idea was presented recently by Vaadia et al. (1995), who analyzed the same data as in this study by the joint-PSTH method (Aertsen et al., 1989). They describe dynamic modulations of coherent activity that appear on a short time scale in relation to behavioral events, even without modification of the average firing ratcs.

Further support for the significance of the neuronal states detected by the HMM was given by Gat and Tishby (1993). Comparison of their results and ours (Abeles et al., 1995) dem- 
onstrates that some of the states that were detected by the two methods share the same firing rates. This result suggests that the recorded neuronal activity is describable by a relatively limited number of states and, thus, that some of the states that were detected in the delay period appear also when the monkey is in other behavioral contexts (e.g., intertrial interval). In addition, Abeles et al. (1995) report that the pairwise correlations between the units varied between the different states that were detected using the $500 \mathrm{msec}$ windows. Thus, a transition from one neuronal state to another is associated not only with concomitant modulations of the firing rates of several units, but also with a change in the statistical structure of the inputs to these cells (e.g., changes in common inputs). This result provides additional support for the global nature of the detected states.

Interestingly, our results suggest that sustained neuronal activity, as observed by methods of PSTH analysis, could be subject to further analysis. For example, several researchers described sustained neuronal activity in frontal, temporal, and parietal cortical areas during delay periods of common behavioral tasks such as delayed-match-tosample, delayed localization, or remembered saccads (Fuster, 1973; Tanji et al., 1980; Wise et al., 1983; Gnat and Andersen, 1988; Miyashita and Chang, 1988; Funahashi et al., 1989, 1993; Motter, 1994; Sawaguchi et al., 1994). This delay activity has been implicated to play a role in working memory, planning of movements, and attention mechanisms. In some of these cases, what appears to be one homogeneous state of sustained delay activity actually may be comprised of several distinct neuronal states with variable transition times between them, as we observe in the later part of the delay. Thus, further analysis of such delay activity using the HMM may provide useful information concerning the internal processes taking place during the delay period.

\section{How can we interpret the neuronal states?}

The most tempting interpretation of our results is that the observed states reflect some higher processes that take place during the delay period. However, some of the neuronal states might be related to uncontrolled motor events. In this study, we monitored only horizontal eye movements. Usually, the monkey fixated the ready light and made only a few horizontal saccades in the first 4 sec of the delay. We cannot eliminate the possibility, however, that these states are related to some unobservable movements or changes in muscular tone during the analyzed period. Even if this is the case, the HMM provides useful information that is unobtainable hy conventional methods. In future studies, it will be interesting to search for motor or behavioral correlates of the neuronal states that are detected by the HMM.

\section{Prediction of the behavior: comparison of the HMM and the NHPM}

The HMM and the NHPM predict the monkey's response correctly in $\sim 90 \%$ of the trials (Table 2) but differ in the time course over which they achieve their predictive power (Fig. 12a). In the early part of the delay, the NHPM gains more predictive power than the HMM. When this early period activity is excluded from the data set, the HMM shows significantly higher predictive power (Fig. 12a). Thus, in the later part of the delay the HMM provides new and useful information that cannot be obtained otherwisethe identification of discrete and stable neuronal states that are specific to the monkey's behavioral condition. When a shuffled data set was subjected to the HMM analysis, the predictive power of the HMM in the later part of the delay fell significantly. Thus, part of this additional predictive power is achieved by the ability of the HMM to detect concomitant firing-rate modulations that are specific to the monkey's behavioral condition.

\section{Two types of underlying dynamics}

Why do the HMM and the NHPM gain predictive power with different time courses? The first second or so after the presenta tion of the visual cue is marked by large firing-rate modulations that are time-locked to the visual cue and are depicted in the PSTH. These modulations appear to be gradual, are consistent from trial to trial and, therefore, are well captured by the NHPM. The HMM, however, assumes that a relatively small number of states with sharp transitions between them describes the entire data set. This assumption makes the HMM less appropriate for modeling the early-period activity (Fig. 13).

Had we recorded from more than six to eight units over more trials, the data might have allowed the estimation of more HMM states. Such an HMM with a large number of hidden states would be similar to an NHPM, but it would have the additional advantage of detecting firing-rate modulations that occur at variable times in different trials. Such a model may surpass the NHPM even in the early period of the trials. If, however, when increasing the number of recorded units the firing-rate modulations of the additional neurons turn out to be uncorrelated with the ones in the recorded set, the NHPM still might be superior.

Our main interest here is in the later period of the trials, when cognitive operations related to memory and planning of movements are likely to occur, and when no time-locked modulations of firing rates can be observed. In this later period, whereas the PSTHs of the units are basically flat, the HMM detects distinct states of activity with sharp transitions between them in individual trials. The higher predictive power of the HMM in this later period, relative to the NHPM, provides strong support for the relevance of the detected states to the monkey's behavior. Further support for the significance of the detected states and the concomitant firing-rate modulations by which they are depicted is provided by the relative robustness of the HMM to the elimination of single units from the data set (Table 3).

Radons et al. (1994) report that the classification performance of a linear classifier, which corresponds to an NHPM, is similar and even better than that of the best HMMs. This result is in accordance with the superior performance of the NHPM that we observe in the early period of the delay, when neuronal activity is driven primarily by the visual stimulus. The results of Radons et al. (1994) suggest that in such periods the local estimates of the firing rates are relatively independent (between different neurons and disjointed time steps) and, therefore, an NHPM provides a more appropriate description of the neuronal data. Our results suggest, however, that neuronal activity in the frontal cortex can display different characteristics; in the later period of the trials, units exhibit highly correlated firing-rate modulations.

Together, our results suggest that different types of network dynamics characterize the early and the later neuronal response in the frontal cortex during the delay. The initial response after the visual stimulus is characterized by smooth and continuous neuronal dynamics that are time-locked to the stimuli and, thus, are explained best by models such as the NHPM. In this period, the states that are detected by the HMM can be viewed as a coarse, discrete approximation of continuous multiunit dynamics. However, in the later period, in which higher processes such as working memory and planning of movements are likely to take place, neuronal activity undergoes state changes that are not time-locked to external events. The underlying neuronal states revealed by the 
HMM analysis in this later period, therefore, may reflect mental states of the monkey.

\section{APPENDIX}

\section{A formal introduction to the HMM}

Under the HMM, neuronal activity is assumed to be governed by a Markov chain of hidden states, whereas in each of these states neurons fire according to independent homogeneous Poisson processes.

Let $X_{t}$ be the state at time step $t(1 \leq t \leq T)$. Define the transition matrix $P$ as $P(i, j)=P\left(X_{\mathrm{t}+1}=j \mid X_{t}=i\right)$, the probability to move from state $i$ to state $j$ at any time step $t$, and the initial probability vector $f_{1}$ as $f_{1}(i)=$ $P\left(X_{1}=i\right)$, the probability that the system starts in state $i$.

Neglecting the unlikely event of simultaneous firing of more than one of the $N$ units at any time step of $1 \mathrm{msec}$, let $Y_{t}=0$ under quiescence and $Y_{t}=$ $i$ if unit $i$ fired at time step $t$. Wc thus have the observed spike train $Y_{1}^{T}=Y_{1}$, $Y_{2}, \ldots, Y_{T}$. Under this model, $Y_{t}$ is assumed to be conditionally independent, given $X_{t}$, of all of the $(X, Y)$ data related to the other time steps.

Define the channel matrix $Q$ as $Q(i, j)=P\left(Y_{r}=j \mid X_{t}=i\right)$, the probability that unit $j$ fires at time step $t$, given that the hidden state is $i$, with $Q(i, 0)$ $=1-\sum_{j=1}^{N} Q(\mathrm{i}, \mathrm{j})$. These probabilities can be viewed as the instantaneous firing rates per time step. For example, a probability $Q(i, j)=5 \times$ $10^{-3} / \mathrm{msec}$ that unit $j$ will fire, given that the hidden state is $i$, corresponds to an average firing rate of 5 spikes/sec.

Summarizing, the model is characterized fully by the parameters $f_{1}, P$, and $Q$. We need to compute in terms of these parameters (1) the likelihood of a given spike train (see Eq. 5), and (2) the conditional probability of each hidden state at any time step, given the observed spike train (see Eq. 6).

To derive these quantitics, we necd to introduce a somewhat claborate numerical structure from which the conditional probabilities of the hidden states and the likelihood of the observations $Y_{1}^{T}$ will follow.

Let

$$
f_{i}(i)=P\left(Y_{1}=y_{1}, Y_{2}=y_{2}, \ldots, Y_{t}=y_{i}, X_{t}=i\right)
$$

be the forward vector, which gives the probability of the partial firing history until time step $t$, with the specification that the hidden state at time $\ell$ is $i$, and let

$$
b_{f}(i)=P\left(Y_{t}=y_{t}, Y_{t+1}=y_{t+1}, \ldots, Y_{T}=y_{T} \mid X_{t}=i\right)
$$

be the backward vector, which gives the probability of the partial firing history from time $t$ until the end of the trial, given that the state at time $t$ is $i$. It is easy to evaluate the forward vectors $f$ and the backward vectors $b$ recursively. Take $f_{\mathrm{i}}$ as given and let $b_{T+1}$ be a vector of ones. Then denoting $\left(y_{l}, y_{l+1}, \ldots, y_{m}\right)$ by $y_{l}^{m}$ and letting $P^{\prime}$ to be the transpose of the matrix $P$,

$$
\begin{aligned}
f_{t+1}(j) & =P\left(Y_{1}^{t}=y_{1}^{t}, Y_{t+1}=y_{t+1}, X_{t+1}=j\right) \\
& =\sum_{i} P\left(Y_{1}^{i}=y_{1}^{t}, X_{t}=i, Y_{t+1}=y_{i+1}, X_{t+1}=j\right) \\
& =\sum_{i} P\left(Y_{1}^{t}=y_{1}^{t}, X_{t}=i\right) P(i, j) Q\left(j, y_{t+1}\right) \\
& =\sum_{i} f_{i}(i) P(i, j) Q\left(j, y_{t+1}\right) \\
& =\left(P^{\prime} f_{i}\right)(j) Q\left(j, y_{t+1}\right),
\end{aligned}
$$

or in words, the probability of a partial history until time step $t+1$ with the specification of the hidden state at that time is expressible using the corresponding terms for time step $t$ and the observed datum $y_{t+1}$. Similarly,

$$
\begin{aligned}
b_{t}(i) & =P\left(Y_{t}^{T}=y_{t}^{T} \mid X_{t}=i\right) \\
& =\sum_{j} P\left(Y_{t}=y_{i}, X_{t+1}=j, Y_{t+1}^{T}=y_{t+1}^{T} \mid X_{t}^{r}=i\right) \\
& =\sum_{j} Q\left(i, y_{t}\right) P(i, j) P\left(Y_{t+1}^{T}=y_{t+1}^{T} \mid X_{t+1}=j\right) \\
& =\sum_{j} Q\left(i, y_{t}\right) P(i, j) b_{t+1}(j) \\
& =\left(P b_{t+1}\right)(i) Q\left(i, y_{t}\right) .
\end{aligned}
$$

From this, we get the likelihood of an observation $y=y_{1}^{T}$ as

$$
\begin{aligned}
f(y)=P\left(Y_{1}^{T}=y_{1}^{T}\right) & =\sum_{i, j} P\left(Y_{1}^{t}=y_{1}^{t}, X_{t}=i, X_{t+1}=j, Y_{t+1}^{T}=y_{t+1}^{T}\right) \\
& =\sum_{i, j} P\left(Y_{1}^{i}=y_{1}{ }_{1}, X_{t}=i\right) P(i, j) P\left(Y_{t+1}^{T}=y_{t+1}^{T} \mid X_{t+1}=j\right) \\
& =\sum_{i, j} f_{t}(i) P(i, j) b_{t+1}(j)=f_{t}^{\prime} P b_{t+1},
\end{aligned}
$$

where $t$ is arbitrary. In particular, taking $t=T$, because $b_{T+1}$ is defined to be a vector of ones, $f(y)=\Sigma_{i} f_{T}(i)$.

These forward and backward vectors allow us to evaluate not only the global likelihood of a given trial, but also the conditional probabilities of the hidden states at any time step $t$, given the entire single trial's spike train:

$$
\begin{aligned}
P\left(X_{t}=i \mid Y_{1}^{T}=y_{1}^{T}\right) & =\frac{P\left(Y_{1}^{t}=y_{1}^{t}, X_{t}=i, Y_{t+1}^{T}=y_{t+1}^{T}\right)}{P\left(Y_{1}^{T}=y_{1}^{T}\right)} \\
& =\sum_{j} \frac{P\left(Y_{1}^{t}=y_{1}^{t}, X_{t}=i, X_{t+1}=j, Y_{t+1}^{T}=y_{t+1}^{T}\right)}{P\left(Y_{1}^{T}=y_{1}^{T}\right)} \\
& =\frac{\sum_{j} f_{i}(i) P(i, j) b_{t+1}(j)}{f(y)} \\
& =\frac{f_{i}(i)\left(P b_{t+1}\right)(i)}{f(y)} .
\end{aligned}
$$

\section{The algorithm for the estimation of the HMM parameters}

The estimation of the transition probability and channel matrices is performed by the Baum-Welch algorithm (Baum, 1970; Jaung, 1984; Rabiner, 1989), which today, from a historical perspective, is viewed as a special case of the expectation maximization (EM) algorithm (Dempster et al., 1977). The rationale behind the EM algorithm is simple. If we assume some modcl parameters ( $P$ and $Q$ in our subject matter), then wc can expect to see approximately $\Sigma_{t} P\left(X_{t}=i, X_{t+1}=j \mid Y_{l}^{T}\right)$ visits to $(i, j)$ in consecutive time steps. Hence, we expect $P^{\text {new }}(i, j)$ (see Eq. 7) to be close to $P(i, j)$, and a similarly defined $Q^{\text {new }}$ (see Eq. 8 ) to be close to $Q$. Maximum likelihood estimators $\hat{P}$ and $\hat{Q}$ have the self-confirmation property $\hat{P}^{\text {new }}=P, \hat{Q}^{\text {new }}=Q$. The EM algorithm replaces $P$ and $Q$ by $P^{\text {new }}$ and $Q^{\text {new }}$ iteratively, reaching a limiting pair $(\hat{P}, \hat{Q})$ that self-confirms. Its stability is related to the fact (Dempster et al., 1977) that the likelihood increases in every iteration.

Letting the sample be composed of the $n$ histories $y^{(1)}=\left(y_{1}^{T}\right)^{(1)}=\left(y_{1}^{(1)}\right.$, $\left.y_{2}^{(1)}, \ldots, y_{T}^{(1)}\right), y^{(2)}=\left(y_{1}^{T}\right)^{(2)}, \ldots, y^{(n)}=\left(y_{1}^{T}\right)^{(n)}$, the update equation for $P^{\text {new }}$ is

$$
\begin{aligned}
P^{\text {ncw }}(i, j) & =\frac{\sum_{k=1}^{n} \sum_{t=1}^{T} P\left(X_{t}=i, X_{t+1}=j \mid Y_{1}^{T}=y^{(k)}\right)}{\sum_{m=1}^{s} \sum_{k=1}^{n} \sum_{i=1}^{T} P\left(X_{t}=i, X_{t+1}=m \mid Y_{1}^{T}=y^{(k)}\right)} \\
& =\frac{\sum_{k} \sum_{t} P\left(X_{t}=i, X_{t+1}=j, Y_{1}^{T}=y^{(k)}\right) / P\left(Y_{1}^{T}=y^{(k)}\right)}{\sum_{m} \sum_{k} \sum_{t} P\left(X_{t}=i, X_{i+1}=m, Y_{1}^{T}=y^{(k)}\right) / P\left(Y_{1}^{T}=y^{(k)}\right)} \\
& =\frac{P^{\text {old }}(i, j) \sum_{k} \sum_{t} f_{i}(i) b_{t+1}(j) / f\left(y^{(k)}\right)}{\sum_{m} P^{\text {old }}(i, m) \sum_{k} \sum_{t} f_{t}(i) b_{t+1}(m) / f\left(y^{(k)}\right)},
\end{aligned}
$$

where $f(y)=P\left(Y_{1}^{T}=y\right)$ is given by Equation 5 . The update equation for $Q^{\text {new }}$ is

$$
\begin{aligned}
& Q^{\mathrm{ncw}}(i, j)=\frac{\sum_{k=1}^{n} \sum_{t=1}^{T} P\left(X_{t}=i, Y_{t}=j \mid Y_{1}^{T}=y^{(k)}\right)}{\sum_{m=0}^{N} \sum_{k=1}^{n} \sum_{t=1}^{T} P\left(X_{t}=i, Y_{t}=m \mid Y_{1}^{T}=y^{(k)}\right)} \\
& \sum_{k} \sum_{,} P\left(X_{t}=i, Y_{1}^{T}=y^{(k)}\right) I_{\left\{y_{t}^{(k)}=j\right.} / P\left(Y_{1}^{T}=y^{(k)}\right) \\
& =\sum_{m} \sum_{k} \sum_{t} P\left(X_{t}=i, Y_{1}^{T}=y^{(k)}\right) I_{\left.\xi_{t}^{(k)}=m\right\}} P\left(Y_{1}^{T}=y^{(k)}\right) \\
& =\frac{\frac{1}{Q^{\text {old }}(i, j)} \sum_{k} \sum_{t} f_{t}(i) I_{\left\langle\psi_{t}^{(k)}=j\right\}} b_{t}(i) / f\left(y^{(k)}\right)}{\sum_{m} \frac{1}{Q^{\text {old }}(i, m)} \sum_{k} \sum_{t} f_{t}(i) I_{\left\langle v_{t}^{(k)}=m\right.} b_{t}(i) / f\left(y^{(k)}\right)}
\end{aligned}
$$


This algorithm allows the simultaneous estimation of the matrices $P$ and $Q$ and the estimation of one of them if the other is given.

\section{A formal presentation of the NHPM}

Under the NHPM, we assume that during every msec $t(1 \leq t \leq 4000)$ at most one neuron fires, and the distribution of the firing neuron under behavioral condition $i$ at time $t$ is given by:

\begin{tabular}{c|ccccc} 
neuron & 1 & 2 & $\ldots$ & $\mathrm{N}$ & none \\
\hline probability & $\lambda_{1}^{(i)}(t)$ & $\lambda_{2}^{(i)}(t)$ & $\ldots$ & $\lambda_{N}^{(i)}(t)$ & $\lambda_{0}^{(i)}(t)=1-\sum_{j=1}^{N} \lambda_{j}^{(i)}(t)$.
\end{tabular}

We further assume that the firing pattern in disjoint time intervals is independent. Our model parameters are the $\lambda$ functions (expressed as firing rates per time step of $1 \mathrm{msec}$ ).

Under these assumptions, the probability under behavioral condition $i$ of an observation $\left(y_{1}, y_{2}, \ldots, y_{4000}\right)$ with $0 \leq y_{\mathrm{t}} \leq N$ is:

$$
f^{(i)}(y)=\prod_{t=1}^{4000} \lambda_{y_{t}}^{(i)}(t)
$$

\section{A note on the prediction procedure}

The intuitive procedure that we use to predict the monkey's behavior (Fig. 11) is a simple example of Bayesian prediction. Under the notation used in Equation 9, letting $K$ be the number of possible behavioral classes, if we are a priori indifferent between the behavioral conditions (in our case, this is reasonable because the number of trials in the different behavioral conditions is similar), then using Bayes formula, the posterior probability of behavioral condition $i$ is

$$
P(i \mid Y=y)=\frac{f^{(i)}(y)}{\sum_{j=1}^{K} f^{(j)}(y)} .
$$

We therefore use a maximal posterior probability measure and predict $i$ to be the behavioral condition for which $P(i \mid Y=y)$ is maximal.

\section{REFERENCES}

Abeles M, Goldstein MH (1977) Multiple spike train analysis. Proc IEEE 65:762-773.

Abeles M, Bergman H, Gat I, Meilijson I, Seidemann E, Tishby N, Vaadia E (1995) Cortical activity flips among quasi stationary states. Proc Natl Acad Sci USA, 92:8616-8620.

Aertsen A, Gerstein GL, Habib MK, Palm G (1989) Dynamics of neuronal firing correlation: modulation of effective connectivity. $J$ Neurophysiol 61:900-917.

Amit DJ (1989) Modeling brain function: the world of attractor neural networks. Cambridge: Cambridge UP.

Baum LE (1970) A maximization technique occurring in the statistical analysis of probabilistic functions of a Markov chains. Ann Math Stat 41:164-171.

Dempster AP, Laird NM, Rubin DB (1977) Maximum likelihood from incomplete data via the EM algorithm. J R Stat Soc B 39:1-38.

Deppisch J, Pawelzik K, Geisel T (1994) Uncovering the synchronization dynamics from correlated neuronal activity quantities assembly formation. Biol Cyber 71:387-399.

Desimone R, Miller E, Chelazzi L, Lueschow A (1995) Multiple memory systems in the visual cortex. In: The cognitive neuroscience (Gazzaniga M, ed), pp 475-486. Cambridge: MIT.

Funahashi S, Bruce C, Goldman-Rakic PS (1989) Mnemonic coding of visual space in the monkey's dorsolateral prefrontal cortex. J Neurophysiol 61:331-349.

Funahashi S, Chafee S, Goldman-Rakic P (1993) Prefrontal neuronal activity in rhesus monkeys performing a delayed anti-saccade task. Nature 365:753-756.

Fuster JM (1973) Unit activity in prefrontal cortex during delayedresponse performance: neural correlates of transient memory. J Neurophysiol 36:61-78.

Fuster JM (1984) Behavioral electrophysiology of the prefrontal cortex. Trends Neurosci 11:408-414.

Fuster JM (1985) The prefrontal cortex and temporal integration. In: Cerebral cortex 4. (Peters A, Jones EG, eds), pp 151-177. New York: Plenum.
Fuster JM (1988) The Prefrontal cortex: anatomy, physiology and neuropsychology of the frontal lobe. New York: Raveni.

Fuster JM, Bauer RH (1974) Visual short-term memory deficit from hypothermia of frontal cortex. Brain Res 81:393-400.

Gat I, Tishby T (1993) Statistical modeling of cell-assemblies activities in associative cortex of behaving monkeys. In: Advances in neural information processing systems (Moody JE, Hamson SJ, Lippmann RP, eds), pp 945-953. San Mateo, CA: Morgan Kaufmann.

Gnat JW, Andersen RA (1988) Memory related motor planning activity in posterior parictal cortcx of monkcy. Exp Brain Res 70:216-220.

Goldman-Rakic PS (1987) Circuitry of primate prefrontal cortex and regulation of behavior by representational memory. In: Handbook of physiology, Sec I, The nervous system (Plum F, ed), pp 373-417. Bethesda: American Physiological Society.

Hopfield J (1982) Neural networks and physical systems with emergent collective abilities. Proc Natl Acad Sci USA 79:2554-2558.

Jaung BH (1984) On the hidden Markov model and dynamic time wrapping for speech recognition-a unified view. AT\&T Bell Lab Tech J 63:1213-1243.

McClurkin JW, Optican LM, Richmond BJ, Gawne TJ (1991) Concurrent processing and complexity of temporal encoded neuronal messages in visual perception. Science 253:675-677.

Milner B, Petrides M (1984) Behavioral effects of frontal lobe lesions. Trends Neurosci 11:403-407.

Miyashita Y, Chang H (1988) Neuronal correlate of pictorial short-term memory in the primate temporal cortex. Nature 331:68-71.

Motter B (1994) Neural correlates of feature selective memory and popout in extrastriate area v4. J Neurosci 14:2190-2199.

Niki H (1974) Prefrontal unit activity during delayed alternation in the monkey. I. Relation to direction of response. Brain Res 68:185-196.

Optican LM, Richmond BJ (1987) Temporal encoding of two dimensional patterns by single units in primates inferior temporal cortex. III. Information theoretic analysis. J Neurophysiol 57:162-178.

Optican LM, Gawne TJ, Richmond BJ, Joseph PJ (1991) Unbiased measure of transmitted information and channel capacity from multivariate neuronal data. Biol Cybern 65:305-310.

Posner M, Petersen S (1990) The attention system of the human brain. In: Annual review of neuroscience (Cowan W, Schooter E, Stevens C, Thompson R, eds), pp 25-42.

Rabiner LR (1989) A tutorial on hidden Markov models and selected applications in speech recognition. Proc IEEE 77:257-85.

Radons G, Becker JP, Dulfer B, Kruger J (1994) Analysis, classification, and coding of multielectrode spike trains with hidden Markov models. Biol Cybern 71:359-373.

Richmond BJ, Optican LM (1987) Temporal encoding of two dimensional patterns by single units in primates inferior temporal cortcx. II. Quantification of response waveform. J Neurophysiol 57:147-161.

Richmond BJ, Optican LM (1990) Temporal encoding of two dimensional patterns by single units in primate primary visual cortex. II. Information transmition. J Neurophysiol 64:370-380.

Richmond BJ, Optican LM, Spitzer H (1990) Temporal encoding of two dimensional patterns by single units in primate primary visual cortex. I. Stimulus response relations. J Neurophysiol 64:351-369.

Sawaguchi T, Goldman Rakic PS (1991) The role of $d_{1}$-dopamine receptor in working memory: local injections of dopamine antagonists into the prefrontal cortex of monkeys performing an oculomotor delayedresponse task. J Neurophysiol 71:515-528.

Seidemann E, Meilijson I, Bergman H, Vaadia E, Abeles M (1994) Ensembles of cortical neurons go through sequence of discrete states which are related to behavioral responses. Soc Neurosci Abstr 20:443.

Tanji J, Taniguchi K, Saga T (1980) Supplementary motor area: neuronal response to motor instructions. J Neurophysiol 43:60-8.

Vaadia E, Haalman I, Abeles M, Bergman H, Prut Y, Slovin H, Aertsen A (1995) Dynamics of neuronal interactions in monkcy cortex in rclation to behavioral events. Nature 373:515-8.

Wise SP, Weinrich M, Mauritz K (1983) Motor aspects of cue-related activity in the premotor cortex of the rhesus monkey. Brain Res 260 : 301-305. 